\title{
Inflationary magnetogenesis, derivative couplings, and relativistic Van der Waals interactions
}

\author{
Massimo Giovannini* \\ Department of Physics, Theory Division, CERN, 1211 Geneva 23, Switzerland INFN, \\ Section of Milan-Bicocca, 20126 Milan, Italy \\ (Received 12 June 2015; published 28 August 2015)
}

\begin{abstract}
When the gauge fields have derivative couplings to scalars, like in the case of the relativistic theory of Van der Waals (or Casimir-Polder) interactions, conformal invariance is broken but the magnetic and electric susceptibilities are not bound to coincide. We analyze the formation of large-scale magnetic fields in slow-roll inflation and find that they are generated at the level of a few hundredths of a nG and over typical length scales between few Mpc and $100 \mathrm{Mpc}$. Using a new time parametrization that reduces to conformal time but only for coincident susceptibilities, the gauge action is quantized while the evolution equations of the corresponding mode functions are more easily solvable. The power spectra depend on the normalized rates of variation of the two susceptibilities (or of the corresponding gauge couplings) and on the absolute value of their ratio at the beginning of inflation. We pin down explicit regions in the parameter space where all the physical requirements (i.e., the backreaction constraints, the magnetogenesis bounds and the naturalness of the initial conditions of the scenario) are jointly satisfied. Weakly coupled initial data are favored if the gauge couplings are of the same order at the end of inflation. Duality is systematically used to simplify the analysis of the wide parameter space of the model.
\end{abstract}

DOI: 10.1103/PhysRevD.92.043521

PACS numbers: 98.80.Cq, 04.62.+v, 98.62.En, 98.80.-k

\section{INTRODUCTION}

Magnetic fields with typical correlation scales exceeding the astronomical unit (i.e., roughly $10^{13} \mathrm{~cm}$ ) permeate the interstellar and intergalactic plasmas which are, in many respects, very similar to the one we can produce in terrestrial experiments $[1,2]$. In spite of this, the origin of large-scale magnetism is still under intense debate both theoretically and observationally [3]. As proposed in the last few years [4] the temperature and the polarization anisotropies of the cosmic microwave background (CMB in what follows) may be magnetized. This observation offers the unique opportunity of direct limits on the large-scale magnetism prior to matter-radiation equality since the large-scale magnetic fields affect directly the initial conditions of the Einstein-Boltzmann hierarchy. The current Planck explorer data can be used to set bounds on largescale magnetic fields [5] as previously done with the WMAP 3-year and 9-year releases (see, respectively, first and second papers of Ref. [5]). The results can be summarized by saying that the WMAP9 $[6,7]$ and Planck data [8] are compatible they are both sensitive to magnetic fields in the $\mathrm{nG}$ range $\left(1 \mathrm{nG}=10^{-9} \mathrm{G}\right)$ for

*massimo.giovannini@cern.ch

Published by the American Physical Society under the terms of the Creative Commons Attribution 3.0 License. Further distribution of this work must maintain attribution to the author(s) and the published article's title, journal citation, and DOI. magnetic spectral indices $n_{B}=\mathcal{O}(1.3)$ using the available temperature and polarization power spectra. ${ }^{1}$

It has been repeatedly argued that large-scale magnetic fields might well be generated during a stage of inflationary expansion. The rationale for this requirement has to do with the correlation scale of the produced field that must be sufficiently large and the onset of the rotation of the protogalaxy. A very promising framework for generating magnetic fields with comoving correlation scales exceeding the $\mathrm{Mpc}$ is represented by models where the gauge fields couple directly to one or more scalar fields (see [9-12] for an incomplete list of references). The scalar fields may coincide with one or (more inflatons) or even with multiple spectator fields. The conventional class of models is based on the following action ${ }^{2}$ :

\footnotetext{
${ }^{1}$ We use here the same conventions employed to assign the curvature power spectra: the scale-invariant magnetic power spectra are realized for $n_{B} \rightarrow 1$ [4,5]. Within these conventions the magnetic power spectrum (i.e., the Fourier transform of the two-point function) has the same dimensions of the magnetic energy density. While other conventions stipulate that the scaleinvariant limit is realized for $n_{B} \rightarrow-3$, in the present paper, the Fourier transform will be consistently assigned for the scalar modes of the geometry and for the magnetic fields implying that $n_{B} \rightarrow 1$ is the scale-invariant limit.

${ }^{2}$ In the notations employed in this paper $Y^{\mu \nu}$ and $\tilde{Y}^{\mu \nu}$ are, respectively, the gauge field strength and its dual; $g=\operatorname{det} g_{\mu \nu}$ is the determinant of the four-dimensional metric with signature mostly minus.
} 


$$
\begin{aligned}
S= & -\frac{1}{16 \pi} \int d^{4} x \sqrt{-g}\left[\lambda(\varphi, \psi) Y_{\alpha \beta} Y^{\alpha \beta}\right. \\
& \left.+\lambda_{\text {pseudo }}(\varphi, \psi) Y_{\alpha \beta} \tilde{Y}^{\alpha \beta}\right],
\end{aligned}
$$

where $\varphi$ and $\psi$ may denote, for instance, a generic inflaton field and a generic spectator field. Various situations can be envisaged and most of them have been investigated in the literature. The presence of $\lambda(\varphi, \psi)$ in Eq. (1.1) is more relevant than the pseudoscalar (axionlike $[13,14]$ ) coupling which will be ignored even if it has been studied by many authors [15-17] in the context of the magnetic field generation. For the amplification of the magnetic field itself the pseudoscalar vertex is not so efficient but it is relevant when one wants to generate magnetic fields whose flux lines are linked or twisted as originally discussed in [18]. The produced Chern-Simons condensate leads to a viable mechanism for baryogenesis via hypermagnetic knots $[18,19]$. These helical fields play also a role in anomalous magnetohydrodynamics where the evolution of the magnetic fields at finite conductivity is analyzed in the presence of anomalous charges [20]. In the collisions of heavy ions this phenomenon is often dubbed chiral magnetic effect [21].

It has been recently argued [22] that the class of models pinned down by Eq. (1.1) can be complemented by further terms:

$$
\begin{aligned}
S= & -\frac{1}{16 \pi} \int d^{4} x \sqrt{-g}\left[\mathcal{M}_{\sigma}^{\rho}(\varphi, \psi) Y_{\rho \alpha} Y^{\sigma \alpha}\right. \\
& \left.-\mathcal{N}_{\sigma}^{\rho}(\varphi, \psi) \tilde{Y}_{\rho \alpha} \tilde{Y}^{\sigma \alpha}\right] .
\end{aligned}
$$

Equation (1.2) leads to unequal electric and magnetic susceptibilities [22]. As a special case Eq. (1.2) includes the typical derivative coupling arising in the relativistic theory of Casimir-Polder and Van der Waals interactions [23]:

$S=-\int d^{4} x \sqrt{-g}\left[g_{1} \partial_{\alpha} \varphi \partial_{\beta} \varphi^{*} Y^{\alpha \rho} Y_{\rho}^{\beta}+g_{2}|\varphi|^{2} Y_{\alpha \beta} Y^{\alpha \beta}\right]$.

Other terms potentially present in Eq. (1.2) (such as $\left.\mathcal{P}_{\rho}^{\sigma} Y_{\sigma \alpha} \tilde{Y}^{\rho \alpha}\right)$ will be neglected even if, as explained above, they might be relevant for the evolution of the magnetic helicity.

The simplest parametrization of the coupling functions $\mathcal{M}_{\rho \sigma}$ and $\mathcal{N}_{\rho \sigma}$ is [22]

$$
\begin{aligned}
& \mathcal{M}_{\rho \sigma}(\varphi)=\lambda_{E}(\varphi) u_{\rho}(\varphi) u_{\sigma}(\varphi), \\
& \mathcal{N}_{\rho \sigma}(\psi)=\lambda_{B}(\psi) \bar{u}_{\rho}(\psi) \bar{u}_{\sigma}(\psi) .
\end{aligned}
$$

Note that $u_{\rho}(\varphi)$ and $\bar{u}_{\rho}(\psi)$ appearing in Eq. (1.4) are the normalized gradients of the corresponding scalar fields but, in the context of a purely hydrodynamical model, they can also play the role of the four-velocities of a relativistic fluid. The full action has been taken to be the sum of the first term of Eq. (1.1) and of the remaining two terms in Eq. (1.3) with the parametrization of Eq. (1.4). In this case the electric and the magnetic susceptibilities are given by

$$
\chi_{E}=\sqrt{\lambda+\frac{\lambda_{E}}{2}}, \quad \chi_{B}=\sqrt{\lambda+\frac{\lambda_{B}}{2}} .
$$

In this framework viable magnetogeneis models can be formulated in different dynamical situations and some of these possibilities have been already swiftly examined [22]. The purpose of the present paper is to undertake a comprehensive analysis of the parameter space of this scenario in terms of the initial conditions and of the evolution of the gauge couplings. To achieve this goal the expressions of $\mathcal{M}_{\rho \sigma}$ and $\mathcal{N}_{\rho \sigma}$ shall be first generalized. The three essential parameters of this class of models are the normalized rate of variation of the electric and magnetic gauge couplings (denoted, respectively, by $F_{E}$ and $F_{B}$ ) and also the initial value of their ratio.

From a pragmatic viewpoint, the whole parameter space can be reduced to the first quadrant of the $\left(F_{B}, F_{E}\right)$ plane where $F_{B}$ and $F_{E}$ are both positive: the other three quadrants can be charted through the systematic use of the duality transformations. ${ }^{3}$ To save time we first impose the backreaction constraints over different scales, then discuss the naturalness of the initial conditions and finally verify if the obtained region of the parameter space satisfies the magnetogensis requirements.

A technical aspect of the present analysis concerns the quantization of the action and the evolution of the related mode functions. This problem can be discussed in the conventional manner by using the conformal time coordinate $\tau$. However the analysis becomes much more transparent in terms of a newly time variable, denoted hereunder by $\eta$, which reduces to the conformal time but only in the case of coincident electric and magnetic gauge couplings.

This paper is organized as follows. In Sec. II we present the generally covariant decomposition of the coupling functions $\mathcal{M}_{\rho \sigma}$ and $\mathcal{N}_{\rho \sigma}$ and study their symmetry properties in the light of the equations of motion. We also show how the obtained parametrization fits within large field and small field inflationary models. In Sec. III we show how the power spectra can be derived by avoiding the standard conformal time parametrization and by expressing the full action in terms of a new time variable directly related to the mismatch between the magnetic and the electric

\footnotetext{
${ }^{3}$ The duality transformations used here are a simple generalization of the standard electromagnetic duality [24]. While in the standard case the electric and the magnetic gauge couplings coincide, in the present situation they differ. See Sec. II for a more detailed discussion.
} 
susceptibilities. The magnetic and electric power spectra are derived in Sec. IV; we shall also analyze how the different regions of the parameter space are transformed under duality and conclude that the most relevant region (from the practical viewpoint) is represented by the first quadrant of the $\left(F_{B}, F_{E}\right)$ plane. In Sec. $\mathrm{V}$ the different portions of the $\left(F_{B}, F_{E}\right)$ plane are scrutinized in the light of the backreaction constraints (and for different initial conditions of the gauge couplings). The allowed region of the parameter space is explicitly obtained and discussed in detail. Section VI contains our concluding remarks and a summary of the main findings.

\section{GENERAL PARAMETRIZATION OF THE COUPLING FUNCTIONS}

The general form of the coupling functions shall now be discussed first in the context of relativistic hydrodynamics and then in the case of conventional inflationary models. This analysis complements the parametrization already outlined in Eq. (1.4).

\section{A. Covariant decompositions}

To begin with let us recall the very well-known covariant decomposition of $\nabla_{\beta} u_{\alpha}$ namely:

$$
\nabla_{\beta} u_{\alpha}=u^{\gamma} u_{\beta} \nabla_{\gamma} u_{\alpha}+\sigma_{\alpha \beta}+\omega_{\alpha \beta}+\frac{\Theta}{3} \mathcal{P}_{\alpha \beta},
$$

where $\Theta=\nabla_{\alpha} u^{\alpha}$ and the projector is defined as $\mathcal{P}_{\alpha \beta}=$ $\left(g_{\alpha \beta}-u_{\alpha} u_{\beta}\right)$. The remaining terms in Eq. (2.1) are given by

$$
\begin{aligned}
& \sigma_{\alpha \beta}= \frac{1}{2}\left(\nabla_{\beta} u_{\alpha}+\nabla_{\alpha} u_{\beta}\right) \\
&-\frac{1}{2} u^{\gamma}\left[\left(\nabla_{\gamma} u_{\alpha}\right) u_{\beta}+\left(\nabla_{\gamma} u_{\beta}\right) u_{\alpha}\right]-\frac{\Theta}{3} \mathcal{P}_{\alpha \beta}, \\
& \omega_{\alpha \beta}=\frac{1}{2}\left(\nabla_{\beta} u_{\alpha}-\nabla_{\alpha} u_{\beta}\right)-\frac{1}{2} u^{\gamma}\left[\left(\nabla_{\gamma} u_{\alpha}\right) u_{\beta}-\left(\nabla_{\gamma} u_{\beta}\right) u_{\alpha}\right] .
\end{aligned}
$$

In what follows, we shall assume that $\omega_{\alpha \beta} \rightarrow 0$. Thus the symmetric coupling tensors $\mathcal{M}_{\rho \sigma}$ and $\mathcal{N}_{\rho \sigma}$ can be written as

$$
\begin{aligned}
\mathcal{M}_{\rho \sigma}= & \lambda_{E} u_{\rho} u_{\sigma}+\frac{\mathcal{D}_{E}}{2 M}\left(\nabla_{\rho} u_{\sigma}+\nabla_{\sigma} u_{\rho}\right) \\
& +\frac{\overline{\mathcal{D}}_{E}}{2 M} u^{\gamma}\left[\left(\nabla_{\gamma} u_{\rho}\right) u_{\sigma}+\left(\nabla_{\gamma} u_{\sigma}\right) u_{\rho}\right], \\
\mathcal{N}_{\rho \sigma}= & \lambda_{B} \bar{u}_{\rho} \bar{u}_{\sigma}+\frac{\mathcal{D}_{B}}{2 M}\left(\nabla_{\rho} \bar{u}_{\sigma}+\nabla_{\sigma} \bar{u}_{\rho}\right) \\
& +\frac{\overline{\mathcal{D}}_{B}}{2 M} \bar{u}^{\gamma}\left[\left(\nabla_{\gamma} \bar{u}_{\rho}\right) \bar{u}_{\sigma}+\left(\nabla_{\gamma} \bar{u}_{\sigma}\right) \bar{u}_{\rho}\right],
\end{aligned}
$$

where $M$ denotes a generic mass scale; $u_{\rho}$ (and $\bar{u}_{\rho}$ ) obey $g^{\rho \sigma} u_{\rho} u_{\sigma}=1$ and $g^{\rho \sigma} \bar{u}_{\rho} \bar{u}_{\sigma}=1$. Notice that we dropped the terms containing $g_{\rho \sigma}$ since it can be reabsorbed by a redefinition of $\lambda$ in Eq. (1.1).

In the analysis of [22] the $\mathcal{D}$ terms have been neglected, i.e., $\mathcal{D}_{E}=\overline{\mathcal{D}}_{E}=0$ and $\mathcal{D}_{B}=\overline{\mathcal{D}}_{B}=0$. All the terms appearing in Eqs. (2.4) and (2.5) will now be considered on equal footing. From Eqs. (1.1) and (1.2) the explicit form of the evolution equations of the gauge fields is

$$
\begin{gathered}
\nabla_{\alpha}\left(\lambda Y^{\alpha \beta}\right)+\frac{1}{2} \nabla_{\alpha} \mathcal{Z}^{\alpha \beta}-\frac{1}{2} \nabla_{\alpha} \mathcal{W}^{\alpha \beta}=4 \pi j^{\beta}, \\
\nabla_{\alpha} \tilde{Y}^{\alpha \beta}=0 .
\end{gathered}
$$

Defining $E^{\alpha \beta \rho \zeta}=\epsilon^{\alpha \beta \rho \zeta} / \sqrt{-g}$ (where $\epsilon^{\alpha \beta \rho \zeta}$ is the total antisymmetric pseudotensor of fourth rank) the two antisymmetric tensors $\mathcal{Z}^{\alpha \beta}$ and $\mathcal{W}^{\alpha \beta}$ are given by

$\mathcal{Z}^{\alpha \beta}=\mathcal{M}_{\sigma}^{\alpha} Y^{\sigma \beta}-\mathcal{M}_{\sigma}^{\beta} Y^{\sigma \alpha}, \quad \mathcal{W}^{\alpha \beta}=E^{\alpha \beta \rho \zeta} \tilde{Y}_{\sigma \zeta} \mathcal{N}_{\rho}^{\sigma}$.

We shall now focus on the case where the background metric is conformally flat:

$$
g_{\mu \nu}(\tau)=a^{2}(\tau) \eta_{\mu \nu}, \quad \mathcal{H}=\frac{a^{\prime}}{a},
$$

where $\eta_{\mu \nu}$ is the Minkowski metric and the prime denotes a derivation with respect to the conformal time coordinate $\tau$. Recalling that the standard Hubble rate is $H=\mathcal{H} / a$, the explicit components of $\mathcal{Z}^{\alpha \beta}$ and $\mathcal{W}^{\alpha \beta}$ are ${ }^{4}$

$$
\begin{gathered}
\mathcal{Z}^{0 i}=-\mathcal{Z}^{i 0}=-\left(\lambda_{E}+\frac{\mathcal{D}_{E}}{M} H\right) \frac{e^{i}}{a^{2}}, \\
\mathcal{Z}^{i j}=-2 H \frac{\mathcal{D}_{E}}{M} \frac{\epsilon^{i j k}}{a^{2}} b_{k}, \\
\mathcal{W}^{0 i}=-\mathcal{W}^{i 0}=2 H \frac{\mathcal{D}_{B}}{M} \frac{e^{i}}{a^{2}}, \\
\mathcal{W}^{i j}=\left(\lambda_{B}+\frac{\mathcal{D}_{B}}{M} H\right) \frac{\epsilon^{i j k}}{a^{2}} b_{k} .
\end{gathered}
$$

\section{B. Equations of motion and duality}

Thanks to Eqs. (2.10) and (2.11), Eqs. (2.6) and (2.7) imply the following form of the equations of motion:

$$
\vec{\nabla} \times\left(\sqrt{\Lambda_{B}} \vec{B}\right)=\partial_{\tau}\left(\sqrt{\Lambda_{E}} \vec{E}\right)+4 \pi \vec{J},
$$

\footnotetext{
${ }^{4}$ We recall that in the conformally flat metric of Eq. (2.9) the gauge field strength can be expressed in terms of the electric and magnetic fields as $Y^{i 0}=e^{i} / a^{2}$ and $Y^{i j}=-\epsilon^{i j k} b_{k} / a^{2}$. The explicit components of $\mathcal{M}_{\rho}^{\sigma}$ and $\mathcal{N}_{\rho}^{\sigma}$ can be explicitly obtained from Eqs. (2.4) and (2.5) and they are $\mathcal{M}_{0}^{0}=\lambda_{E}, \mathcal{N}_{0}^{0}=\lambda_{B}$, $\mathcal{M}_{i}^{j}=\mathcal{D}_{E} H \delta_{i}^{j} / M$ and $\mathcal{N}_{i}^{j}=\mathcal{D}_{B} H \delta_{i}^{j} / M$.
} 


$$
\begin{gathered}
\vec{\nabla} \times\left(\frac{\vec{E}}{\sqrt{\Lambda_{E}}}\right)+\partial_{\tau}\left(\frac{\vec{B}}{\sqrt{\Lambda_{B}}}\right)=0, \\
\vec{\nabla} \cdot\left(\frac{\vec{B}}{\sqrt{\Lambda_{B}}}\right)=0, \quad \vec{\nabla} \cdot\left(\sqrt{\Lambda_{E}} \vec{E}\right)=4 \pi \rho,
\end{gathered}
$$

where we have introduced the following rescaled variables:

$$
\begin{gathered}
\vec{B}=a^{2} \sqrt{\Lambda_{B}} \vec{b}, \quad \vec{E}=a^{2} \sqrt{\Lambda_{E}} \vec{e} \\
\Lambda_{B}=\lambda+\frac{\lambda_{B}}{2}+\frac{\mathcal{D}_{B}}{2 M} H+\frac{\mathcal{D}_{E}}{M} H, \\
\Lambda_{E}=\lambda+\frac{\lambda_{E}}{2}+\frac{\mathcal{D}_{E}}{2 M} H+\frac{\mathcal{D}_{B}}{M} H .
\end{gathered}
$$

Concerning Eqs. (2.15), (2.16) and (2.17) three comments are in order:

(i) in the homogenous case the terms containing $\overline{\mathcal{D}}_{E}$ and $\overline{\mathcal{D}}_{B}$ do not contribute to the evolution of the gauge fields;

(ii) in the simultaneous limit $\mathcal{D}_{B} \rightarrow 0$ and $\mathcal{D}_{E} \rightarrow 0$ we have that $\Lambda_{B}=\lambda+\lambda_{B} / 2$ and that $\Lambda_{E}=\lambda+\lambda_{E} / 2$, as previously established;

(iii) if we transform $\lambda_{E} \rightarrow \lambda_{B}, \mathcal{D}_{B} \rightarrow \mathcal{D}_{E}$ and $\mathcal{D}_{E} \rightarrow \mathcal{D}_{B}$ then $\Lambda_{E} \rightarrow \Lambda_{B}$ (and vice versa).

The susceptibilities $\chi_{E}$, and $\chi_{B}$ are defined as $\chi_{E}=\sqrt{\Lambda_{E}}$ and $\chi_{B}=\sqrt{\Lambda_{B}}$. The corresponding gauge couplings are instead the inverse of the susceptibilities ${ }^{5}$

$$
g_{E}=\sqrt{4 \pi / \Lambda_{E}}, \quad g_{B}=\sqrt{4 \pi / \Lambda_{B}} .
$$

The mismatch between the two susceptibilities (i.e., $\left.f=\chi_{E}^{2} / \chi_{B}^{2}=\Lambda_{E} / \Lambda_{B}\right)$ determines an effective refractive index affecting the evolution of the canonical fields.

In Eqs. (2.12) and (2.14) $\vec{J}$ and $\rho$ denote, respectively, the rescaled current density and the rescaled charge density. In what follows we shall analyze the amplification of the gauge fields from vacuum fluctuations so we shall set the sources to zero. There are however different possible initial conditions like the ones stipulating that initially a globally neutral plasma is present, namely $\rho=0$ with $\vec{J}$ given by the Ohmic current of the plasma. This possibility has been investigated in the conventional case [25] but shall not be specifically analyzed here.

An important aspect that will be systematically used to simplify the discussion of the parameter space concerns the symmetries of the model. Neglecting the sources, Eqs. (2.12), (2.13) and (2.14) are invariant under the generalized duality transformation [22] that coincides with

\footnotetext{
${ }^{5}$ Note that in the limit $\lambda_{B}=\lambda_{E} \rightarrow 0$ and $\mathcal{D}_{E}=\mathcal{D}_{B} \rightarrow 0, \chi_{E}$ and $\chi_{B}$ coincide and the same happens for the gauge couplings, i.e., $g_{E}=g_{B}=\sqrt{4 \pi / \lambda}$.
}

the conventional duality [24] in the case of coincident electric and magnetic gauge couplings. When the gauge couplings are exchanged and inverted (i.e., $g_{E} \rightarrow 1 / g_{B}$ and $\left.g_{B} \rightarrow 1 / g_{E}\right)$ the generalized duality transformation stipulates that Eqs. (2.12), (2.13) and (2.14) keep exactly the same form provided $\vec{E} \rightarrow-\vec{B}$ and $\vec{B} \rightarrow \vec{E}$.

\section{Coupling functions and scalar fields}

We shall now examine the form of the coupling functions when the four-velocity is related to the covariant gradients of a scalar field (i.e., either the inflaton or some other spectator field); in this case from the general considerations discussed above we shall have

$$
\begin{aligned}
\mathcal{M}_{\rho \sigma}(\varphi)= & \mathcal{B}_{1}(\varphi) \nabla_{\rho} \varphi \nabla_{\sigma} \varphi+\frac{\mathcal{B}_{2}(\varphi)}{2}\left(\nabla_{\rho} \nabla_{\sigma} \varphi+\nabla_{\sigma} \nabla_{\rho} \varphi\right) \\
& +\frac{\mathcal{B}_{3}(\varphi)}{2} \nabla^{\gamma} \varphi\left(\nabla_{\rho} \varphi \nabla_{\gamma} \nabla_{\sigma} \varphi+\nabla_{\sigma} \varphi \nabla_{\gamma} \nabla_{\rho} \varphi\right),
\end{aligned}
$$

$$
\begin{aligned}
\mathcal{N}_{\rho \sigma}(\psi)= & \mathcal{C}_{1}(\psi) \nabla_{\rho} \psi \nabla_{\sigma} \psi+\frac{\mathcal{C}_{2}(\psi)}{2}\left(\nabla_{\rho} \nabla_{\sigma} \psi+\nabla_{\sigma} \nabla_{\rho} \psi\right) \\
& +\frac{\mathcal{C}_{3}(\psi)}{2} \nabla^{\gamma} \psi\left(\nabla_{\rho} \psi \nabla_{\gamma} \nabla_{\sigma} \psi+\nabla_{\sigma} \psi \nabla_{\gamma} \nabla_{\rho} \psi\right)
\end{aligned}
$$

As discussed before $\mathcal{M}_{\rho \sigma}$ and $\mathcal{N}_{\rho \sigma}$ do not have to depend on the same field. For illustration two different scalar fields (i.e., $\varphi$ and $\psi$ ) have been used in Eqs. (2.19) and (2.20). The general form of Eqs. (2.19) and (2.20) suggests that it is always possible to select the arbitrary coupling functions in such a way that the electric and magnetic gauge couplings depend on the scale factor. During the inflationary phase their analytic dependence can always be written as

$$
\begin{array}{r}
g_{E}(a)=\bar{g}_{E}\left(\frac{a}{a_{i}}\right)^{F_{E}}, \quad g_{B}(a)=\bar{g}_{B}\left(\frac{a}{a_{i}}\right)^{F_{B}}, \\
f(a)=f_{i}\left(\frac{a}{a_{i}}\right)^{F}, \quad f_{i}=\frac{\bar{g}_{B}^{2}}{\bar{g}_{E}^{2}}=\frac{\bar{\Lambda}_{B}}{\bar{\Lambda}_{E}},
\end{array}
$$

where $F_{E}, F_{B}$ and $F$ denote, respectively, the normalized rates of variation, i.e., ${ }^{6}$

$$
F_{E}=\frac{\partial \ln g_{E}}{\partial \ln a}, \quad F_{B}=\frac{\partial \ln g_{B}}{\partial \ln a}, \quad F=\frac{\partial \ln f}{\partial \ln a},
$$

and, by definition, $F=2\left(F_{B}-F_{E}\right)$. In Eqs. (2.21)-(2.22) $a_{i}$ denotes the value of the scale factor at the onset of the evolution of the gauge couplings; this moment will be taken

\footnotetext{
${ }^{6} \mathrm{We}$ recall that in this paper $\ln$ denotes the natural logarithm while log denotes the common logarithm.
} 
to coincide, for simplicity, with the onset of the inflationary phase. ${ }^{7}$ Recalling Eq. (2.18), the parametrization of Eqs. (2.21) and (2.22) implies that $\chi_{E}(a) \propto a^{-F_{E}}$ and $\chi_{B} \propto a^{-F_{B}}$.

The parameter counting of Eqs. (2.21) and (2.22) goes as follows. There are nominally four parameters in Eqs. (2.21) and (2.22), namely the rates $F_{E}$ and $F_{B}$ and the corresponding amplitudes, i.e., $\bar{g}_{E}$ and $\bar{g}_{B}$. These four parameters can be identified with $F_{E}, F_{B}, f_{i}$ and with the initial value of one of the gauge couplings (be it either $\bar{g}_{B}$ or $\bar{g}_{E}$ ). Neither $\bar{g}_{B}$ or $\bar{g}_{E}$ appear alone in the final electric and magnetic power spectra but only combined in $f_{i}$ which can be different from one and, in principle, also very large or very small depending on the specific model under consideration. On top of these three parameters there is also the total duration of the inflationary phase and, possibly, the overall duration of the pumping action of the gauge couplings.

\section{Power-law backgrounds}

Before proceeding further, the parametrization of Eqs. (2.21) and (2.22) shall now be justified in the context of specific scenarios. This step is, in a sense, superfluous given the arbitrariness of the coupling functions. However it might be interesting to have this point treated in more depth. In what follows we shall assume the validity of the standard inflationary evolution and examine different specific models. The power law backgrounds are interesting insofar as they are exactly solvable. In this case it is immediately evident that the coupling functions must have an exponential or quasiexponential form if we want to justify the validity of the parametrization of Eqs. (2.21) and (2.22). In power-law backgrounds $\epsilon=-\dot{H} / H^{2}=1 / \alpha$ and

$$
\begin{aligned}
\dot{\varphi}^{2} & =\frac{2 \alpha \bar{M}_{P}^{2}}{t^{2}}, \quad \varphi=\varphi_{0}+\sqrt{\frac{2}{\epsilon}} \bar{M}_{P} \ln \left(t / t_{i}\right), \\
a(t) & =\left(t / t_{i}\right)^{\alpha},
\end{aligned}
$$

where $t_{i}$ denotes the initial cosmic time of the inflationary expansion. It can also happen that the susceptibilities do not depend on the inflaton but only on some spectator field that evolves during a quasi-de Sitter stage of expansion. In this case the evolution equation of $\psi$ can be solved even exactly like in the case of an exponential potential. If the field $\psi$ evolves in an exact power-law background, its evolution will be given as

$$
\begin{aligned}
\psi & =\psi_{0}+\mathcal{K}_{1} \bar{M}_{P} \ln \left(t / t_{i}\right), \\
W(\psi) & =W_{0} e^{-\mathcal{K}_{2}\left(\psi-\psi_{0}\right) / \bar{M}_{P},}
\end{aligned}
$$

where $\mathcal{K}_{1} \mathcal{K}_{2}=2$ and $W_{0} \mathcal{K}_{2}=H_{i}^{2} \bar{M}_{P}^{2} \epsilon(\epsilon-3)$. With this information, the explicit couplings can be explicitly

\footnotetext{
${ }^{7}$ If this identification is not made there will be a supplementary parameter fixing the origin of the evolution of the gauge couplings in comparison with the onset of the inflationary phase.
}

constructed and they will fall within the parametrization of Eqs. (2.21) and (2.22).

In the conventional case the coupling functions are exponentials [9-11]. If we make the same choice here, $\lambda(\varphi)=Q_{0} \exp \left[q_{0}\left(\varphi-\varphi_{0}\right)\right]$ sets the overall normalization while the other couplings can be parametrized as

$$
\begin{array}{ll}
\mathcal{B}_{1}(\varphi) & =Q_{1} \frac{e^{q_{1}\left(\varphi-\varphi_{0}\right) / \bar{M}_{P}}}{H_{i}^{2} \bar{M}_{P}^{2}}, \quad \mathcal{B}_{2}(\varphi)=Q_{2} \frac{e^{q_{2}\left(\varphi-\varphi_{0}\right) / \bar{M}_{P}}}{H_{i}^{2} \bar{M}_{P}}, \\
\mathcal{B}_{3}(\varphi) & =Q_{3} \frac{e^{q_{3}\left(\varphi-\varphi_{0}\right) / \bar{M}_{P}}}{H_{i}^{4} \bar{M}_{P}^{3}}
\end{array}
$$

Exactly the same parametrization can be envisaged for $\mathcal{C}_{1}(\psi), \mathcal{C}_{2}(\psi)$ and $\mathcal{C}_{3}(\psi)$ where we choose, for simplicity, $\psi=\varphi$ :

$$
\begin{array}{ll}
\mathcal{C}_{1}(\varphi)=P_{1} \frac{e^{p_{1}\left(\varphi-\varphi_{0}\right) / \bar{M}_{P}}}{H_{i}^{2} \bar{M}_{P}^{2}}, & \mathcal{C}_{2}(\varphi)=P_{2} \frac{e^{p_{2}\left(\varphi-\varphi_{0}\right) / \bar{M}_{P}}}{H_{i}^{2} \bar{M}_{P}}, \\
\mathcal{C}_{3}(\varphi)=P_{3} \frac{e^{p_{3}\left(\varphi-\varphi_{0}\right) / \bar{M}_{P}}}{H_{i}^{4} \bar{M}_{P}^{3}}
\end{array}
$$

Equations (2.26) and (2.27) extend the class of models of Refs. [9-12] to the case of derivative interactions obtained through the present construction.

The explicit form of the couplings of Eqs. (2.26) and (2.27) together with the background evolution of Eq. (2.24) leads to the parametrization of Eqs. (2.21) and (2.22). While more complicated dynamical situations are certainly possible (and should be separately investigated) we shall now argue that the monotonic evolution of the gauge couplings is realized in various explicit scenarios. Let us assume, as above, $\psi=\varphi$ and also set $\mathcal{B}_{1}(\varphi)=\mathcal{B}_{2}(\varphi)=$ $\mathcal{B}_{3}(\varphi)=0$ together with $\mathcal{C}_{3}(\varphi)=0$. The explicit form of the susceptibilities, in the case of exponential couplings, will then $\mathrm{be}^{8}$

$$
\begin{aligned}
\Lambda_{E}(\tau)= & Q_{0}\left(-\frac{\tau}{\tau_{*}}\right)^{\sqrt{2 \epsilon} q_{0} /(\epsilon-1)} \\
& +P_{2} \sqrt{2 \epsilon}\left(-\frac{\tau}{\tau_{*}}\right)^{\sqrt{2 \epsilon}\left(p_{2}-\sqrt{2 \epsilon}\right) /(\epsilon-1)}, \\
\Lambda_{B}(\tau)= & Q_{0}\left(-\frac{\tau}{\tau_{*}}\right)^{\sqrt{2 \epsilon} q_{0} /(\epsilon-1)}+P_{1} \epsilon\left(-\frac{\tau}{\tau_{*}}\right)^{\sqrt{2 \epsilon}\left(p_{1}-\sqrt{2 \epsilon}\right) /(\epsilon-1)} \\
& +\frac{P_{2}}{2} \sqrt{2 \epsilon}(1-\epsilon)\left(-\frac{\tau}{\tau_{*}}\right)^{\sqrt{2 \epsilon}\left(p_{2}-\sqrt{2 \epsilon}\right) /(\epsilon-1)}
\end{aligned}
$$

Depending on the relative hierarchy of $q_{0}, p_{1}$ and $p_{2}$ the paranetrization of Eqs. (2.21) and (2.22) can be easily

\footnotetext{
${ }^{8}$ The connection between cosmic and conformal time can always be written as $\left(t / t_{i}\right)=\left(-\tau / \tau_{*}\right)^{1 /(1-\alpha)}$ where $\tau_{*}=t_{i} /$ $(\alpha-1)$.
} 
recovered after some putative time related to $\tau_{*}$. This parametrization may also lead to more complicated situations where $f(\tau)$ may even have a nonmonotonic behavior. However it is sufficient to suppose that $p_{2} \gg$ $q_{0}$ and $p_{1} \gg p_{2} \gg q_{0}$ to obtain

$\chi_{E}(a)=\bar{\chi}_{E}\left(\frac{a}{a_{*}}\right)^{-F_{E}}, \quad \chi_{B}(a)=\bar{\chi}_{B}\left(\frac{a}{a_{*}}\right)^{-F_{B}}$,

where $F_{E}=\sqrt{\epsilon / 2}\left(p_{2}-\sqrt{2 \epsilon}\right) /(\epsilon-1)$ and $F_{B}=$ $\sqrt{\epsilon / 2}\left(p_{1}-\sqrt{2 \epsilon}\right) /(\epsilon-1)$; moreover $\bar{\chi}_{E}^{2}=\sqrt{2 \epsilon} P_{2}$ and $\bar{\chi}_{B}^{2}=\epsilon P_{1}$. The initial value of the susceptibilities and the relative strength of the couplings can be tuned by changing $P_{1}$ and $P_{2}$.

\section{E. Slow-roll models}

The previous discussion can be easily generalized to the case of generic slow-roll evolution. The simplest situation in this respect is given by the case where all the undetermined functions vanish except two of them. Let us therefore set $\mathcal{B}_{1}(\varphi)=\mathcal{B}_{2}(\varphi)=\mathcal{B}_{3}(\varphi)=0$ and also $\mathcal{C}_{3}(\varphi)=$ $\mathcal{C}_{1}(\varphi)=0$. Given a certain inflationary potential we can always define

$$
\mathcal{I}\left(\varphi_{i}, \varphi\right)=\frac{1}{\bar{M}_{P}^{2}} \int_{\varphi_{i}}^{\varphi} \frac{W}{W_{, \varphi}} d \varphi .
$$

The coupling functions can then be fixed as

$$
\begin{aligned}
\mathcal{C}_{2}(\varphi) & =\frac{6}{W_{, \varphi}} e^{-2 F_{E} \mathcal{I}\left(\varphi_{i}, \varphi\right)}\left[e^{-2\left(F_{B}-F_{E}\right) \mathcal{I}\left(\varphi_{i}, \varphi\right)}-1\right], \\
\lambda(\varphi) & =e^{-2 F_{E} \mathcal{I}\left(\varphi_{i}, \varphi\right)}\left[2 e^{-2\left(F_{B}-F_{E}\right) \mathcal{I}\left(\varphi_{i}, \varphi\right)}-1\right] .
\end{aligned}
$$

Another possibility is to set $\mathcal{C}_{2}(\varphi)=\mathcal{C}_{3}(\varphi)=0$ and, as before $\mathcal{B}_{1}(\varphi)=\mathcal{B}_{2}(\varphi)=\mathcal{B}_{3}(\varphi)=0$. The values of $\lambda(\varphi)$ and $\mathcal{C}_{1}(\varphi)$ are given, in this case, by

$$
\begin{aligned}
\lambda(\varphi) & =e^{-2 F_{E} \mathcal{I}\left(\varphi_{i}, \varphi\right)}, \\
\mathcal{C}_{1}(\varphi) & =\frac{3}{W \epsilon} e^{-2 F_{E} \mathcal{I}\left(\varphi_{i}, \varphi\right)}\left[e^{-2\left(F_{B}-F_{E}\right) \mathcal{I}\left(\varphi_{i}, \varphi\right)}-1\right] .
\end{aligned}
$$

Equations (2.32) and (2.33) imply that the susceptibilities can be written, in a unified notation, as

$\chi_{B}^{2}=\lambda+\frac{\mathcal{C}_{1}(\varphi)}{2} \dot{\varphi}^{2}-\mathcal{C}_{2}(\varphi)\left[H \dot{\varphi}+\frac{W_{, \varphi}}{2}\right]=\bar{\chi}_{B}^{2}\left(\frac{a}{a_{i}}\right)^{-2 F_{B}}$,

$\chi_{E}^{2}=\lambda+\mathcal{C}_{2}(\varphi) H \dot{\varphi}=\bar{\chi}_{E}^{2}\left(\frac{a}{a_{i}}\right)^{-2 F_{E}}$,

where we recall that in the slow-roll approximation $\left(a / a_{i}\right)=\exp \left[-\mathcal{I}\left(\varphi_{i}, \varphi\right)\right]$; furthermore the slow-roll relations have been used where appropriate. The same analysis leading to Eq. (2.34) can be repeated when all the $\mathcal{C}_{i}(\varphi)$ are equal to zero while, on top of $\lambda(\varphi)$, the only two nonvanishing coupling functions are either $\mathcal{B}_{1}(\varphi)$ or $\mathcal{B}_{2}(\varphi)$.

It is obvious that depending on the model the relation between $\left(a / a_{i}\right)$ and $\varphi$ will be a bit different but still the general parametrization of Eqs. (2.21) and (2.22) will remain valid. In the case of simple monomial potentials we have $W=M^{4} \phi^{p}$ and therefore we shall have that

$$
\left(\frac{a}{a_{i}}\right)=\exp \left[-\frac{\varphi^{2}-\varphi_{i}^{2}}{2 p \bar{M}_{P}^{2}}\right] .
$$

Other typical models can be analyzed like the case of small field and hybrid models where the potential can be written as $W(\varphi)=M^{4}\left(1 \pm \kappa \varphi^{p}\right)$ (where the plus corresponds to the hybrid models while the minus to the small field models). In the case of $R^{2}$ models the potential, in the Einstein frame, is given by

$$
W(\varphi)=\frac{3 M^{2} \bar{M}_{P}^{2}}{4}\left(1-e^{-\sqrt{2 / 3} \varphi / \bar{M}_{P}}\right)^{2},
$$

where we defined, for practical reasons, $\Phi=\sqrt{2 / 3} \varphi / \bar{M}_{P}$. Using the evolution equations we obtain

$\Phi=\ln \left[2 M\left(t_{*}-t\right) / 3\right], a=a_{*} \exp 3\left[\Phi-e^{\Phi}\right] / 4$.

In cosmic time the scale factor reads

$$
a(t)=\left[M\left(t_{*}-t\right)\right]^{3 / 4} e^{-M\left(t_{*}-t\right) / 2},
$$

where $t_{*}$ denotes in practice the final time of the inflationary expansion. All the specific analyses discussed before can be easily applied also to this case with just one caveat. To obtain the specific equations relating the cosmic and the conformal time parametrization we must expand the above equations in the limit $t \ll t_{*}$. The result of this simple expansion can be written as

$$
a \simeq a_{*} x_{*}^{3 / 4} e^{-x_{*} / 2} e^{\left[2 x-3\left(x / x_{*}\right)\right] / 4},
$$

where $x=M t$ and $x_{*}=M t_{*}$. Thanks to the previous expression we can easily deduce the relation of $t$ to $\tau$ by recalling that $a(\tau) d \tau=d t$. In summary we have demonstrated that while the most general parametrization of the coupling functions may lead to nonmonotonic susceptibilities, after some initial transient time the monotonic behavior can be recovered. The parametrization of Eqs. (2.21) and (2.22) is therefore fully justified and it encompasses a variety of models.

\section{QUANTIZATION AND POWER SPECTRA}

The problem is greatly simplified by abandoning the conformal time and by rescaling the canonical variables. 
This transformation leaves the action invariant and simplifies the evolution of the mode functions.

\section{A. Time reparametrization of the action and quantization}

The main idea is to use a new time parametrization in the action and a new set of rescaled vector potentials. In time-dependent (conformally flat) backgrounds and in the Coulomb gauge (i.e., $Y_{0}=0$ and $\vec{\nabla} \cdot \vec{Y}=0$ ) that is preserved (unlike the Lorentz gauge condition) under a conformal rescaling of the metric the action is

$$
\begin{aligned}
S= & \frac{1}{2} \int d \tau d^{3} x\left\{\vec{A}^{\prime 2}+\left(\frac{\chi_{E}^{\prime}}{\chi_{E}}\right)^{2} \vec{A}^{2}\right. \\
& \left.-2 \frac{\chi_{E}^{\prime}}{\chi_{E}} \vec{A} \cdot \vec{A}^{\prime}-\frac{\chi_{B}^{2}}{\chi_{E}^{2}} \partial_{i} \vec{A} \cdot \partial^{i} \vec{A}\right\},
\end{aligned}
$$

where $^{9} \vec{A}=\sqrt{\Lambda_{E} /(4 \pi)} \vec{Y}$. We have assumed that $\chi_{E}$ and $\chi_{B}$ are only dependent on the conformal time coordinate $\tau$. In terms of the canonical momentum conjugate to $\vec{A}$ the canonical Hamiltonian is simply given by [22]

$$
\begin{aligned}
H_{A}(\tau) & =\frac{1}{2} \int d^{3} x\left[\vec{\pi}^{2}+2 \frac{\chi_{E}^{\prime}}{\chi_{E}} \vec{\pi} \cdot \vec{A}+\frac{\partial_{i} \vec{A} \cdot \partial^{i} \vec{A}}{f}\right], \\
\vec{\pi} & =\vec{A}^{\prime}-\frac{\chi_{E}^{\prime}}{\chi_{E}} \vec{A} .
\end{aligned}
$$

We can now change the time parametrization in the action of Eq. (3.1)

$$
\tau \rightarrow \eta=\eta(\tau), \quad d \tau=\sqrt{f(\eta)} d \eta,
$$

and simultaneously redefine the vector potential:

$$
\vec{A}(\tau, \vec{x}) \rightarrow \mathcal{A}(\eta, \vec{x})=\frac{\vec{A}[\tau(\eta), \vec{x}]}{f^{1 / 4}[\tau(\eta)]} .
$$

Ultimately $\overrightarrow{\mathcal{A}}$ can be directly expressed in terms of $\vec{Y}$ as

$$
\begin{aligned}
\overrightarrow{\mathcal{A}}(\eta, \vec{x}) & =\sqrt{\frac{\Lambda_{E}(\eta)}{4 \pi \sqrt{f(\eta)}}} \vec{Y}(\eta, \vec{x}) \\
& =\frac{\sqrt{4} \Lambda_{E}(\eta) \Lambda_{B}(\eta)}{\sqrt{4 \pi}} \vec{Y}(\eta, \vec{x})=\frac{\vec{Y}(\eta, \vec{x})}{\sqrt{g_{E}(\eta) g_{B}(\eta)}},
\end{aligned}
$$

where the second and third equalities follow, respectively, from the mutual relations among the different susceptibilities and from the relation between each susceptibility and the corresponding gauge coupling. Using Eqs. (3.3) and (3.4) the action (3.1) becomes

\footnotetext{
${ }^{9}$ The $1 / \sqrt{4 \pi}$ is purely conventional and its presence comes from the factor $16 \pi$ included in the initial gauge action.
}

$$
\begin{aligned}
S= & \frac{1}{2} \int \frac{1}{2} \int d \eta d^{3} x\left\{\partial_{\eta} \overrightarrow{\mathcal{A}} \cdot \partial_{\eta} \overrightarrow{\mathcal{A}}+\left[\frac{\left(\sqrt{\chi_{E} \chi_{B}}\right)^{\bullet}}{\sqrt{\chi_{E} \chi_{B}}}\right]^{2} \overrightarrow{\mathcal{A}}^{2}\right. \\
& \left.-2 \frac{\left(\sqrt{\chi_{E} \chi_{B}}\right)^{\bullet}}{\sqrt{\chi_{E} \chi_{B}}} \partial_{\eta} \overrightarrow{\mathcal{A}} \cdot \overrightarrow{\mathcal{A}}-\partial_{i} \overrightarrow{\mathcal{A}} \cdot \partial^{i} \overrightarrow{\mathcal{A}}\right\},
\end{aligned}
$$

where the thick overdot (or bullet) denotes a derivation with respect to $\eta$. From the action (3.6) the new canonical Hamiltonian and the corresponding canonical momenta are now given by

$$
\begin{gathered}
H_{\mathcal{A}}(\eta)=\frac{1}{2} \int d^{3} x\left[\vec{\Pi}^{2}+2 \frac{\left(\sqrt{\chi_{E} \chi_{B}}\right)^{\bullet}}{\sqrt{\chi_{E} \chi_{B}}} \vec{\Pi} \cdot \overrightarrow{\mathcal{A}}+\partial_{i} \overrightarrow{\mathcal{A}} \cdot \partial^{i} \overrightarrow{\mathcal{A}}\right], \\
\vec{\Pi}(\eta, \vec{x})=\partial_{\eta} \overrightarrow{\mathcal{A}}-\frac{\left(\sqrt{\chi_{E} \chi_{B}}\right)^{\bullet}}{\sqrt{\chi_{E} \chi_{B}}} \overrightarrow{\mathcal{A}}
\end{gathered}
$$

The Fourier mode expansion for the canonical fields

$$
\begin{aligned}
\vec{\Pi}(\vec{x}, \eta) & =\frac{1}{(2 \pi)^{3 / 2}} \int d^{3} k \vec{\Pi}_{\vec{k}}(\eta) e^{-i \vec{k} \cdot \vec{x}}, \\
\vec{A}(\vec{x}, \eta) & =\frac{1}{(2 \pi)^{3 / 2}} \int d^{3} k \overrightarrow{\mathcal{A}}_{\vec{k}}(\eta) e^{-i \vec{k} \cdot \vec{x}}
\end{aligned}
$$

implies that the canonical Hamiltonian (3.7) can be rewritten as

$$
\begin{aligned}
H_{A}(\eta)= & \frac{1}{2} \int d^{3} k\left[\vec{\Pi}_{\vec{k}} \cdot \vec{\Pi}_{-\vec{k}}+\frac{\left(\sqrt{\chi_{E} \chi_{B}}\right)^{\bullet}}{\sqrt{\chi_{E} \chi_{B}}}\right. \\
& \left.\times\left(\vec{\Pi}_{\vec{k}} \cdot \overrightarrow{\mathcal{A}}_{-\vec{k}}+\vec{\Pi}_{-\vec{k}} \cdot \overrightarrow{\mathcal{A}}_{\vec{k}}\right)+k^{2} \overrightarrow{\mathcal{A}}_{\vec{k}} \cdot \overrightarrow{\mathcal{A}}_{-\vec{k}}\right] .
\end{aligned}
$$

From Eq. (3.10) the corresponding equations or motion are

$$
\begin{array}{r}
\partial_{\eta} \overrightarrow{\mathcal{A}}_{\vec{k}}=\vec{\Pi}_{\vec{k}}+\sqrt{g_{B} g_{E}}\left(\frac{1}{\sqrt{g_{B} g_{E}}}\right) \cdot \overrightarrow{\mathcal{A}}_{\vec{k}}, \\
\partial_{\eta} \vec{\Pi}_{\vec{k}}=-k^{2} \overrightarrow{\mathcal{A}}_{\vec{k}}-\sqrt{g_{B} g_{E}}\left(\frac{1}{\sqrt{g_{B} g_{E}}}\right) \vec{\Pi}_{\vec{k}},
\end{array}
$$

where we have used the explicit relations connecting the susceptibilities to the gauge couplings. The duality transformation exchanges the canonical fields and the conjugate momenta so that Eqs. (3.11) and (3.12) go one into the other by virtue of the following transformation:

$$
\begin{aligned}
& g_{E}(\eta) \rightarrow \frac{1}{g_{B}(\eta)}, \quad g_{B}(\eta) \rightarrow \frac{1}{g_{E}(\eta)}, \\
& \vec{\Pi}_{\vec{k}}(\eta) \rightarrow-k \overrightarrow{\mathcal{A}}_{\vec{k}}(\eta), \quad \overrightarrow{\mathcal{A}}_{\vec{k}}(\eta) \rightarrow \frac{\vec{\Pi}_{\vec{k}}(\eta)}{k} .
\end{aligned}
$$


Promoting the canonical fields to quantum operators (i.e., $\mathcal{A}_{i} \rightarrow \hat{\mathcal{A}}_{i}$ and $\pi_{i} \rightarrow \hat{\pi}_{i}$ ) the following (equal time) commutation relations (in units $\hbar=c=1$ ) must hold:

$$
\begin{aligned}
{\left[\hat{\mathcal{A}}_{i}\left(\vec{x}_{1}, \eta\right), \hat{\Pi}_{j}\left(\vec{x}_{2}, \eta\right)\right] } & =i \Delta_{i j}\left(\vec{x}_{1}-\vec{x}_{2}\right), \\
\Delta_{i j}\left(\vec{x}_{1}-\vec{x}_{2}\right) & =\int \frac{d^{3} k}{(2 \pi)^{3}} e^{i \vec{k} \cdot\left(\vec{x}_{1}-\vec{x}_{2}\right)} P_{i j}(k),
\end{aligned}
$$

where $P_{i j}(k)=\left(\delta_{i j}-k_{i} k_{j} / k^{2}\right)$. The function $\Delta_{i j}\left(\vec{x}_{1}-\vec{x}_{2}\right)$ is the transverse generalization of the Dirac delta function ensuring that both $\vec{E}$ and $\vec{A}$ are divergenceless. The field operators can then be expanded in terms of the corresponding mode functions

$$
\begin{aligned}
\hat{\mathcal{A}}_{i}(\eta, \vec{x})= & \int \frac{d^{3} k}{(2 \pi)^{3 / 2}} \sum_{\alpha} e_{i}^{(\alpha)}(k)\left[\bar{F}_{k}(\eta) \hat{a}_{k, \alpha} e^{-i \vec{k} \cdot \vec{x}}\right. \\
& \left.+\bar{F}_{k}^{*}(\eta) \hat{a}_{k, \alpha}^{\dagger} e^{i \vec{k} \cdot \vec{x}}\right], \\
\hat{\Pi}_{i}(\eta, \vec{x})= & \int \frac{d^{3} k}{(2 \pi)^{3 / 2}} \sum_{\alpha} e_{i}^{(\alpha)}(k)\left[\bar{G}_{k}(\eta) \hat{a}_{k, \alpha} e^{-i \vec{k} \cdot \vec{x}}\right. \\
& \left.+\bar{G}_{k}^{*}(\eta) \hat{a}_{k, \alpha}^{\dagger} e^{i \vec{k} \cdot \vec{x}}\right],
\end{aligned}
$$

where $\bar{F}_{k}(\eta)$ and $\bar{G}_{k}(\eta)$ are the mode functions in the $\eta$ parametrization.

\section{B. General solutions for the mode functions}

As a consequence of the indetermination relations, Eqs. (3.15) and (3.16) imply that the mode functions must obey the equations derived previously and the Wronskian normalization condition:

$$
\bar{F}_{k}(\eta) \bar{G}_{k}^{*}(\eta)-\bar{F}_{k}^{*}(\eta) \bar{G}_{k}(\eta)=i .
$$

From Eqs. (3.11) and (3.12), $\bar{F}_{k}(\eta)$ and $\bar{G}_{k}(\eta)$ can be shown to satisfy the following pair of equations:

$$
\begin{gathered}
\frac{d \bar{F}_{k}}{d \eta}=\bar{G}_{k}+\sqrt{g_{B} g_{E}}\left(\frac{1}{\sqrt{g_{B} g_{E}}}\right)^{\cdot} \bar{F}_{k}, \\
\frac{d \bar{G}_{k}}{d \eta}=-k^{2} \bar{F}_{k}-\sqrt{g_{B} g_{E}}\left(\frac{1}{\sqrt{g_{B} g_{E}}}\right)^{\cdot} \bar{G}_{k} .
\end{gathered}
$$

Combining Eqs. (3.18) and (3.19) we obtain two decoupled equations:

$$
\begin{gathered}
\frac{d^{2} \bar{F}_{k}}{d \eta^{2}}+\left[k^{2}-\sqrt{g_{B} g_{E}}\left(\frac{1}{\sqrt{g_{B} g_{E}}}\right)^{\bullet \cdot}\right] \bar{F}_{k}=0, \\
\frac{d^{2} \bar{G}_{k}}{d \eta^{2}}+\left[k^{2}-\frac{\left(\sqrt{g_{B} g_{E}}\right)^{\bullet \bullet}}{\sqrt{g_{B} g_{E}}}\right] \bar{G}_{k}=0 .
\end{gathered}
$$

Equations (3.20) and (3.21) can also be written in terms of the susceptibilities $\chi_{E}$ and $\chi_{B}$ by simply taking into account that, as already mentioned, $g_{E}=\sqrt{4 \pi / \chi_{E}}$ and $g_{B}=$ $\sqrt{4 \pi / \chi_{B}}$. Equations (3.18)-(3.19) and (3.20)-(3.21) can be directly derived from the mode functions obtainable from the quantization in conformal time. Indeed, using the recipe

$$
\begin{aligned}
& \frac{d \tau}{\sqrt{f[\tau(\eta)]}}=d \eta, \quad F_{k}(\tau)=f^{1 / 4}[\eta(\tau)] \bar{F}_{k}[\eta(\tau)], \\
& G_{k}[\eta(\tau)]=f^{-1 / 4}[\eta(\tau)] \bar{G}_{k}[\eta(\tau)],
\end{aligned}
$$

Equations (3.18)-(3.19) become immediately

$F_{k}{ }^{\prime}=G_{k}+\frac{\chi_{E}{ }^{\prime}}{\chi_{E}} F_{k}, \quad G_{k}{ }^{\prime}=-\frac{k^{2}}{f} F_{k}-\frac{\chi_{E}{ }^{\prime}}{\chi_{E}} G_{k}$,

where the prime denotes a derivation with respect to $\tau$; Eqs. (3.23) coincide with the ones derived in [22].

The general solutions of Eqs. (3.20) and (3.21) can be obtained either exactly (i.e., by solving the evolution in exact terms) or approximately (i.e., in different asymptotic limits). In a large class of physical situations the pump fields of Eqs. (3.20) and (3.21) are expected to decay for $|\eta| \rightarrow \infty$ faster than $1 / \eta$ (see, however, the end of this subsection for a different situation). The solution of Eq. (3.20) can then be obtained as

$\bar{F}_{k}(\eta)=\frac{1}{\sqrt{2 k}} e^{ \pm i k \eta}, \quad k^{2} \gg\left|\sqrt{g_{E} g_{B}}\left(\frac{1}{\sqrt{g_{B} g_{E}}}\right)^{\bullet \cdot}\right|$,

$$
\begin{aligned}
\bar{F}_{k}(\eta) & =\frac{D_{k}^{(1)}}{\sqrt{g_{E} g_{B}}}+\frac{D_{k}^{(2)}}{\sqrt{g_{E} g_{B}}} \int^{\eta} d \eta_{1} g_{E}\left(\eta_{1}\right) g_{B}\left(\eta_{1}\right), \\
k^{2} & \ll\left|\sqrt{g_{E} g_{B}}\left(\frac{1}{\sqrt{g_{B} g_{E}}}\right)\right|,
\end{aligned}
$$

where $D_{k}^{(1)}$ and $D_{k}^{(2)}$ are the two arbitrary constants that must be fixed from the boundary conditions. Recalling Eq. (3.18), Eqs. (3.24)-(3.25) also determine the explicit form of $\bar{G}_{k}(\eta)$. Alternatively Eq. (3.21) can be directly solved with the same strategy leading to Eqs. (3.24) and (3.25).

\section{Exact solutions with asymmetric gauge couplings}

According to Eqs. (3.20)-(3.21) and using the generalized parametrization of Eqs. (2.21) and (2.22), the evolution of the mode functions obeys

$$
\begin{array}{r}
\frac{d^{2} \bar{F}_{k}}{d \eta^{2}}+\left[k^{2}-\frac{\sigma^{2}-1 / 4}{\eta^{2}}\right] \bar{F}_{k}=0, \\
\frac{d^{2} \bar{G}_{k}}{d \eta^{2}}+\left[k^{2}-\frac{(\sigma-1)^{2}-1 / 4}{\eta^{2}}\right] \bar{G}_{k}=0,
\end{array}
$$


whose solution is is given by

$\bar{F}_{k}(\eta)=\frac{\mathcal{N}}{\sqrt{2 k}} \sqrt{-k \eta} H_{\sigma}^{(1)}(-k \eta), \quad \sigma=\frac{1-2 F_{E}}{2\left(1+F_{B}-F_{E}\right)}$,

$$
\bar{G}_{k}(\eta)=-\mathcal{N} \sqrt{\frac{k}{2}} \sqrt{-k \eta} H_{\sigma-1}^{(1)}(-k \eta),
$$

where $|\mathcal{N}|=\sqrt{\pi / 2}$. The case $F_{E}=F_{B}+1$ is a bit special since we have that $f=\bar{f}\left(\tau / \tau_{i}\right)^{2}$. But in this situation the susceptibilities can be parametrized, in general terms, as ${ }^{10}$

$$
\begin{aligned}
\chi_{E}(\eta) & =\bar{\chi}_{E} e^{\alpha_{E}\left(\eta+\eta_{i}\right) / \eta_{*}}, \quad \chi_{B}(\eta)=\bar{\chi}_{B} e^{\alpha_{B}\left(\eta+\eta_{i}\right) / \eta_{*}}, \\
f(\eta) & =\bar{f} e^{2\left(\alpha_{E}-\alpha_{B}\right)\left(\eta+\eta_{i}\right) / \eta_{*}} .
\end{aligned}
$$

The evolution equation for $\bar{F}_{k}(\eta)$ can be written as

$$
\frac{d^{2} \bar{F}_{k}}{d \eta^{2}}+\left[k^{2}-\frac{\left(\alpha_{E}+\alpha_{B}\right)^{2}}{4 \eta_{*}^{2}}\right] \bar{F}_{k}=0
$$

and the solution of the previous equation is

$$
\bar{F}_{k}(\eta)=\frac{1}{\sqrt{2 \Omega_{k}}} e^{-i \Omega_{k}\left(\eta+\eta_{i}\right)}, \Omega_{k}=\sqrt{k^{2}-\frac{\left(\alpha_{E}+\alpha_{B}\right)}{4 \eta_{*}^{2}}} .
$$

If $k \eta_{*} \gg 1$ then $\Omega_{k} \simeq k$ while in the opposite limit $\Omega_{k} \simeq i\left|\alpha_{E}+\alpha_{B}\right| /\left(2 \eta_{*}\right)$. Recalling the expression of $\bar{G}_{k}$ we have that

$$
\bar{G}_{k}=-i \sqrt{\frac{\Omega_{k}}{2}}\left[1-\frac{i\left(\alpha_{E}+\alpha_{B}\right)}{2 \Omega \eta_{*}}\right] e^{-i \Omega_{k}\left(\eta+\eta_{*}\right)} .
$$

Note that in the limit $k \eta_{*} \ll 1$ we have that $\bar{G}_{k}(\eta) \rightarrow 0$ and $P_{E}(k, \eta) \rightarrow 0$ provided $\alpha_{E}$ and $\alpha_{B}$ are both positive semidefinite. Conversely, in the same limit,

$$
\left|\bar{F}_{k}(\eta)\right|^{2}=\frac{4 \eta_{*}}{\left|\alpha_{E}+\alpha_{B}\right|} e^{\left|\alpha_{E}+\alpha_{B}\right|\left(\eta+\eta_{i}\right) / \eta_{*}} .
$$

\section{Correlation functions}

In the $\eta$ parametrization the correlation functions of the electric and of the magnetic fields can be swiftly computed. It is sufficient to recall that the canonical fields can be directly expressed in terms of the comoving electric and magnetic fields, i.e., $\vec{E}$ and $\vec{B}$ :

\footnotetext{
${ }^{10}$ In this specific case the relation between $\tau$ and $\eta$ is exponential since $\eta$ is the logarithm of $\tau$, i.e., $\ln \left(-\tau / \tau_{i}\right)=$ $-\sqrt{\bar{f}}\left(\eta / \tau_{i}\right)$ and $\eta_{i}=\tau_{i} / \sqrt{\bar{f}}$.
}

$\vec{E}(\eta, \vec{x})=-\frac{\vec{\Pi}(\eta, \vec{x})}{\sqrt{4} f(\eta)}, \quad \vec{B}(\eta, \vec{x})=\vec{\nabla} \times\left[\frac{\overrightarrow{\mathcal{A}}(\eta, \vec{x})}{\sqrt{4} f(\eta)}\right]$.

In terms of the mode decomposition, the comoving electric and magnetic fields are

$$
\begin{aligned}
\hat{B}_{i}(\eta, \vec{x})= & -\frac{i \epsilon_{m n i}}{(2 \pi)^{3 / 2} \sqrt{4} f(\eta)} \sum_{\alpha} \int d^{3} k k_{m} e_{n}^{(\alpha)} \\
& \times\left[\bar{F}_{k}(\eta) \hat{a}_{\vec{k}, \alpha} e^{-i \vec{k} \cdot \vec{x}}-\bar{F}_{k}^{*}(\eta) \hat{a}_{\vec{k}, \alpha}^{\dagger} e^{i \vec{k} \cdot \vec{x}}\right], \\
\hat{E}_{i}(\eta, \vec{x})= & -\frac{1}{(2 \pi)^{3 / 2} \sqrt{4} f(\eta)} \sum_{\alpha} \int d^{3} k e_{i}^{(\alpha)} \\
& \times\left[\bar{G}_{k}(\eta) \hat{a}_{\vec{k}, \alpha} e^{-i \vec{k} \cdot \vec{x}}+\bar{G}_{k}^{*}(\eta) \hat{a}_{\vec{k}, \alpha}^{\dagger} e^{i \vec{k} \cdot \vec{x}}\right] .
\end{aligned}
$$

In terms of the mode functions, the Fourier components of the field operators $\hat{B}_{i}(\vec{x}, \tau)$ and $\hat{E}_{i}(\vec{x}, \tau)$ are, respectively,

$$
\begin{gathered}
\hat{B}_{i}(\eta, \vec{q})=-\frac{i}{\sqrt{4} f(\eta)} \epsilon_{m n i} \sum_{\alpha} e_{n}^{(\alpha)} q_{m} \\
\times\left[\hat{a}_{\vec{q}, \alpha} \bar{F}_{q}(\eta)+\hat{a}_{-\vec{q}, \alpha}^{\dagger} \bar{F}_{q}^{*}(\eta)\right], \\
\hat{E}_{i}(\eta, \vec{q})=\frac{1}{\sqrt{4} f(\eta)} \sum_{\alpha} e_{i}^{(\alpha)}\left[\hat{a}_{\vec{q}, \beta} \bar{G}_{q}(\eta)+\hat{a}_{-\vec{q}, \beta}^{\dagger} \bar{G}_{q}^{*}(\eta)\right] .
\end{gathered}
$$

The correlators in Fourier space are then given by

$$
\left\langle B_{i}(\eta, \vec{k}) B_{j}(\eta, \vec{p})\right\rangle=\frac{2 \pi^{2}}{k^{3}} P_{B}(k, \eta) P_{i j}(k) \delta^{(3)}(\vec{k}+\vec{p}),
$$

$\left\langle E_{i}(\eta, \vec{k}) E_{j}(\eta, \vec{p})\right\rangle=\frac{2 \pi^{2}}{k^{3}} P_{E}(k, \eta) P_{i j}(k) \delta^{(3)}(\vec{k}+\vec{p})$,

where $P_{i j}(k)=\left(\delta_{i j}-k_{i} k_{j} / k^{2}\right)$ and where the power spectra are

$$
\begin{aligned}
& P_{B}(k, \eta)=\frac{k^{5}}{2 \pi^{2} a^{4}(\eta) \sqrt{f(\eta)}}\left|\bar{F}_{k}(\eta)\right|^{2}, \\
& P_{E}(k, \eta)=\frac{k^{3}}{2 \pi^{2} a^{4}(\eta) \sqrt{f(\eta)}}\left|\bar{G}_{k}(\eta)\right|^{2} .
\end{aligned}
$$

Note that, as a consequence of the duality symmetry, the magnetic and the electric power spectra are interchanged when $g_{E} \rightarrow 1 / g_{B}$ and $g_{B} \rightarrow 1 / g_{E}$. In this case $\bar{G}_{k} \rightarrow-k \bar{F}_{k}$ and $\bar{F}_{k} \rightarrow \bar{G}_{k} / k$ so that $P_{B}(k, \eta) \rightarrow P_{E}(k, \eta)$ and vice versa. 
It is very important to remark, for the following discussion, that the definition of the power spectrum of Eqs. (3.39) and (3.40) is particularly suitable since $P_{B}(k, \eta)$ and $P_{E}(k, \eta)$ have both dimensions of an energy density. Consequently we shall measure the amplitude of the power spectrum either in critical units or in $\mathrm{nG}^{2}$. We stress that this occurrence is not only mathematically useful but also physically sound: if the power spectrum is defined according to the present strategy it is evident, as it has to be, that the magnetic power spectrum is also (up to an irrelevant numerical factor) the magnetic energy density per logarithmic interval of wavenumbers (or logarithmic interval of frequency).

\section{POWER SPECTRA AND INITIAL DATA}

\section{A. General form of the power spectra}

The power spectra depend on the initial data of the electric and magnetic susceptibilities (or, which is the same, of the electric and magnetic gauge couplings). Two complementary possibilities can be envisaged. The first possibility stipulates that $f\left(a_{i}\right)=f_{i}=1$, i.e., $\chi_{E}\left(a_{i}\right)=\chi_{B}\left(a_{i}\right)$ : the susceptibilities are initially equal and then they diverge so that, depending on the number of efolds, they will still be different at the end of inflation. The second possibility is the one where $f\left(a_{f}\right)=f_{f}=1$; in this second case $\chi_{E}\left(a_{f}\right)=\chi_{B}\left(a_{f}\right)$, i.e., the susceptibilities are initially very different but they become equal at the end of inflation. In this second case $f\left(a_{i}\right) \gg 1$. The various intermediate situations (stipulating that initially there is an arbitrary mismatch between the susceptibilities) will fall between the patterns of the two limiting cases $f_{i}=1$ and $f_{f}=1$.

With this distinction in mind, and recalling Eqs. (2.21)(2.22) and (2.23), the solution of the mode function of Eqs. (3.27) implies that the magnetic power spectrum becomes

$$
\begin{aligned}
P_{B}(k, a, \sigma) & =H^{4} \mathcal{Q}_{B}(\sigma, \mu) f_{i}^{|\sigma|-1}\left(\frac{k}{a H}\right)^{5-2|\sigma|}\left(\frac{a}{a_{i}}\right)^{2 \mu(|\sigma|-1)}, \\
\mathcal{Q}_{B}(\sigma, \mu) & =\frac{\Gamma^{2}(|\sigma|)}{\pi^{3}} 2^{2|\sigma|-3}|1+\mu|^{2|\sigma|-1}
\end{aligned}
$$

where, for practical reasons, the following notation has been employed:

$$
\sigma=\frac{1-2 F_{E}}{1+F_{B}-F_{E}}, \quad \mu=\frac{F}{2}=F_{B}-F_{E} .
$$

Similarly thanks to Eq. (3.28) the electric power spectrum is

$$
\begin{aligned}
P_{E}(k, a, \sigma)= & H^{4} \mathcal{Q}_{E}(\sigma, \mu) f_{i}^{|\sigma-1|-1} \\
& \times\left(\frac{k}{a H}\right)^{5-2|\sigma-1|}\left(\frac{a}{a_{i}}\right)^{2 \mu(|\sigma-1|-1)}, \\
\mathcal{Q}_{E}(\sigma, \mu)= & \frac{\Gamma^{2}(|\sigma-1|)}{\pi^{3}} 2^{2|\sigma-1|-3}|1+\mu|^{2|\sigma-1|-1} .
\end{aligned}
$$

Note that in the plane $\left(F_{B}, F_{E}\right)$ there is a singular trajectory, namely $1+F_{B}-F_{E}=0$ where $\sigma$ diverges. This singularity is not physical and stems from the fact that for $F_{E}=F_{B}+1$ the gauge couplings evolve exponentially in $\eta$.

An essential feature of Eqs. (3.39) and (3.40) is that the electric and the magnetic power spectra both have dimensions of an energy density in Fourier space and this is related to the fact that the scale-invariant limit of the spectra occurs for $n_{B} \rightarrow 1$ and $n_{E} \rightarrow 1$. As already mentioned we shall measure the magnetic power spectrum either in critical units or in $\mathrm{nG}^{2}$. Another way of measuring the amplitudes of the power spectra will be to use critical units and, in this case, the magnetic and electric power spectra can be expressed as

$$
\begin{aligned}
& \Omega_{B}\left(k, a, \sigma, \mu, f_{i}\right) \\
& \quad=\frac{8 \pi^{2}}{3} \epsilon \mathcal{A}_{\mathcal{R}} \mathcal{Q}_{B}(\sigma, \mu) f_{i}^{|\sigma|-1}\left(\frac{k}{a H}\right)^{5-2|\sigma|}\left(\frac{a}{a_{i}}\right)^{2 \mu(|\sigma|-1)},
\end{aligned}
$$

$$
\begin{aligned}
& \Omega_{E}\left(k, a, \sigma, \mu, f_{i}\right) \\
& \quad=\frac{8 \pi^{2}}{3} \epsilon \mathcal{A}_{\mathcal{R}} \mathcal{Q}_{E}(\sigma, \mu) f_{i}^{|\sigma-1|-1}\left(\frac{k}{a H}\right)^{5-2|\sigma-1|}\left(\frac{a}{a_{i}}\right)^{2 \mu(|\sigma-1|-1)} .
\end{aligned}
$$

Notice that in Eqs. (4.4) and (4.5) we traded $H / M_{P}$ for $\mathcal{A}_{\mathcal{R}}$, i.e., the amplitude of the power spectrum of curvature perturbations at the pivot scale $k_{p}=0.002 \mathrm{Mpc}^{-1}$; note also that the slow-roll parameter $\epsilon$ appears in the final expressions.

The magnetic and the electric spectral indices can be easily deduced from Eqs. (4.1)-(4.3) and from Eqs. (4.4)-(4.5) and they are

$$
n_{B}=6-2|\sigma|, \quad n_{E}=6-2|\sigma-1| .
$$

If a certain hierarchy between the gauge couplings is imposed ab initio for some theoretical consideration, then $f_{i}$ is fixed. Similarly $f_{i}$ can also be fixed by tuning $f\left(a_{f}\right)$ at a certain specific value, be it $f_{f}$ : in this case $f_{f}=f_{i}\left(a_{f} / a_{i}\right)^{2 \mu}$. In the conformal time parametrization the spatial gradient leads to a term $k / \sqrt{f}$ which can be either blueshifted or redshifted depending on $\sqrt{f}$. Naively we would require that $\sqrt{f}>1$ to avoid a light speed that is larger than one. This observation, however, depends on the time parametrization. For instance in the cosmic time parametrization we have that the physical frequency is given by $k / a(t)$; if we would apply the same tenets we would exclude the interesting possibility of contracting universes. In the specific cases discussed below, the cases $\sqrt{f} \ll 1$ are phenomenologically disfavored and are anyway excluded for more physical reasons. Let us finally note that to fix $f_{i}$ or $f_{f}$ is in general different. However in the 
framework of the monotonic parametrization of Eqs. (2.21) and (2.22) the value of $f_{f}$ is uniquely determined as a function to $f_{i}$ through the total number of inflationary efolds, i.e., $\ln \left(f_{f} / f_{i}\right)=2\left(F_{B}-F_{E}\right) N_{t}$. Therefore, for the purposes of the concrete discussion, the boundary conditions can be fixed either in terms of $f_{i}$ or in terms of $f_{f}$.

\section{B. Physical regions of the parameter space}

The six different regions of the parameter space in the $\left(F_{E}, F_{B}\right)$ plane are illustrated in Fig. 1 where on the horizontal and vertical axes we report, respectively, $F_{B}$ and $F_{E}$, i.e., the rates of variation of $g_{B}$ and $g_{E}$. The different regions appearing in Fig. 1 correspond to six different dynamical situations that can be summarized for short as follows:

(I) In the first region the gauge couplings are both increasing, i.e., $F_{B} \geq 0$ and $F_{E} \geq 0$; furthermore also $f$ increases since $F_{B} \geq F_{E}$.

(II) In the second region the gauge couplings are also increasing but since $F_{E} \geq F_{B}$ we have that $F<0$ and $f$ is bound to decrease.

(III) In the third region $F_{B}<0$ and $F_{E}>0$ (leading to $F<0)$.

(IV) In the fourth region both gauge couplings are decreasing, (i.e., $F_{B}<0$ and $F_{E}<0$ ) while $F_{B}<$ $F_{E}$ and consequently $F<0$.

(V) The gauge couplings are also decreasing simultaneously (i.e., $F_{E}<0$ and $F_{B}<0$ ) but in this region $F>0$ (i.e., $f$ increases).

(VI) In the sixth and last region $F_{E}<0$ and $F_{B}>0$ implying $F>0$ and the corresponding growth of $f$.

In regions (I) and (II) the gauge couplings are both increasing; in regions (IV) and (V) the gauge couplings are both decreasing (but at a different rate); finally in regions (III) and (VI) one of the two gauge couplings is increasing while the other is decreasing. Recalling Eq. (4.2) the spectral indices can be directly expressed in terms of $F_{E}$ and $F_{B}$ :

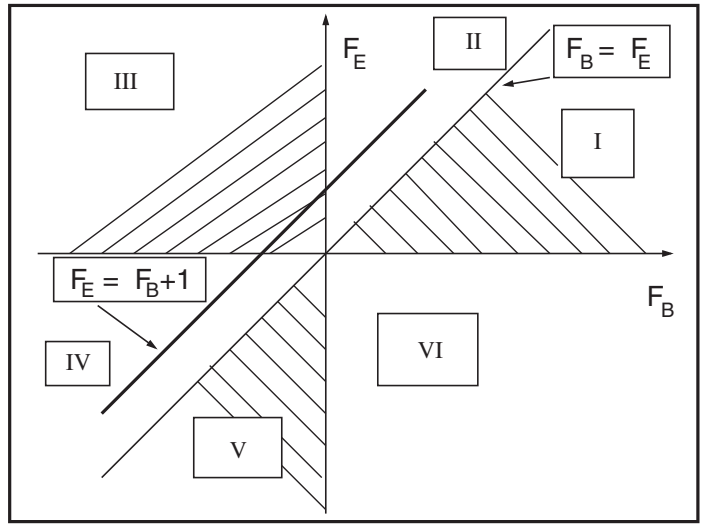

FIG. 1. The parameter space of the model is schematically illustrated in terms of the normalized rates of variation of the gauge couplings.

$$
\begin{aligned}
& n_{B}\left(F_{B}, F_{E}\right)=6-\left|\frac{1-2 F_{E}}{\left(1+F_{B}-F_{E}\right)}\right|, \\
& n_{E}\left(F_{B}, F_{E}\right)=6-\left|\frac{1+2 F_{B}}{\left(F_{E}-F_{B}-1\right)}\right|
\end{aligned}
$$

Thanks to Eq. (4.7) it is immediate to show that, under duality,

$F_{E} \rightarrow-F_{B}, \quad F_{B} \rightarrow-F_{E}, \quad n_{B} \rightarrow n_{E}, \quad n_{E} \rightarrow n_{B}$.

Furthermore, recalling Eq. (4.2), under duality $\sigma \rightarrow \bar{\sigma}=$ $1-\sigma$.

The magnetic power spectra solely expressed in terms of $F_{B}$ and $F_{E}$ become then

$$
\begin{aligned}
P_{B}\left(k, F_{B}, F_{E}, f_{i}\right)= & H^{4} \mathcal{Q}_{B}\left(F_{B}, F_{E}\right) f_{i}^{\left[4-n_{B}\left(F_{B}, F_{E}\right)\right] / 2} \\
& \times\left(\frac{k}{a H}\right)^{n_{B}\left(F_{B}, F_{E}\right)-1} \mathcal{N}_{B}\left(N_{t}, F_{B}, F_{E}\right), \\
\mathcal{Q}_{B}\left(F_{B}, F_{E}, f_{i}\right)= & \frac{\Gamma^{2}\left[\left(6-n_{B}\left(F_{B}, F_{E}\right)\right) / 2\right]}{\pi^{3}} 2^{3-n_{B}\left(F_{B}, F_{E}\right)} \\
& \times\left|1+F_{B}-F_{E}\right|^{5-n_{B}\left(F_{B}, F_{E}\right)} . \\
\mathcal{N}_{B}\left(F_{B}, F_{E}\right)= & e^{N_{t}\left(F_{B}-F_{E}\right)\left[4-n_{B}\left(F_{B}, F_{E}\right)\right]} .
\end{aligned}
$$

As a function of $F_{B}$ and $F_{E}$ the electric power spectra become instead

$$
\begin{aligned}
P_{E}\left(k, N_{t}, F_{B}, F_{E}\right)= & H^{4} \mathcal{Q}_{E}\left(F_{B}, F_{E}\right) f_{i}^{\left[4-n_{E}\left(F_{B}, F_{E}\right)\right] / 2} \\
& \times\left(\frac{k}{a H}\right)^{n_{E}\left(F_{B}, F_{E}\right)-1} \mathcal{N}_{E}\left(N_{t}, F_{B}, F_{E}\right), \\
\mathcal{Q}_{E}\left(F_{B}, F_{E}\right)= & \frac{\Gamma^{2}\left[\left(6-n_{E}\left(F_{B}, F_{E}\right)\right) / 2\right]}{\pi^{3}} 2^{3-n_{E}\left(F_{B}, F_{E}\right)} \\
& \times\left|1+F_{B}-F_{E}\right|^{5-n_{E}\left(F_{B}, F_{E}\right)}, \\
\mathcal{N}_{E}\left(N_{t}, F_{B}, F_{E}\right)= & e^{N_{t}\left(F_{B}-F_{E}\right)\left[4-n_{E}\left(F_{B}, F_{E}\right)\right]},
\end{aligned}
$$

where $n_{B}\left(F_{B}, F_{E}\right)$ and $n_{E}\left(F_{B}, F_{E}\right)$ are now given in Eq. (4.7). With the same strategy the corresponding critical fractions of Eqs. (4.4) and (4.5) can be easily expressed in the $\left(F_{B}, F_{E}\right)$ plane. Let us finally mention that Eqs. (4.9) and (4.10) go one into the other thanks to a duality transformation. This important property, already mentioned in the previous section, is now explicitly verifiable at the level of the final results and thanks to Eq. (4.8).

\section{Dualizing the regions of the parameter space}

In each region of Fig. 1, depending on the values of $F_{E}$, $F_{B}$ and $F$, the power spectra have different slopes and different amplitude. This aspect is illustrated in Fig. 2 where we analyze the parameter space from the viewpoint of the slope of the power spectra. The shaded regions of Fig. 2 correspond to different spectral indices and different values 


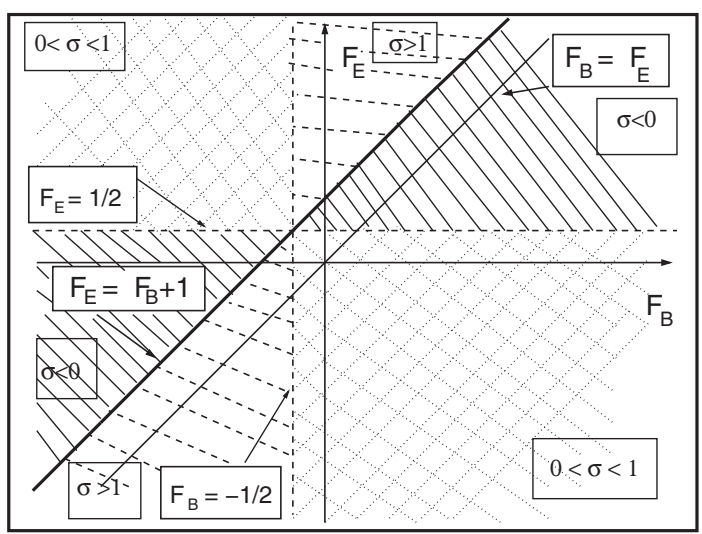

FIG. 2. Dependence of the power spectra on the rates of the gauge couplings.

of $\sigma$. Under duality the various regions are interchanged. Consider, for instance, the first quadrant of Fig. 2 where the gauge couplings are both increasing, i.e., $F_{E}>0$ and $F_{B}>0$. In this region we have

$$
\begin{aligned}
0 & <F_{E}<1 / 2, \quad 0<\sigma<1, \\
1 / 2 & <F_{E}<F_{B}+1, \quad \sigma<0, \\
F_{E} & >F_{B}+1, \quad \sigma>1 .
\end{aligned}
$$

For each region of Eq. (4.11) the spectral indices have a different functional dependence upon $F_{E}$ and $F_{B}$. In Table I the spectral indices in the first quadrant are summarized. Notice that the electric spectral index is always expressible in terms of $n_{B}$ but the relation changes from region to region. In Fig. 2 there are peculiar lines dividing the different shaded areas. The straight line $F_{E}=F_{B}+1$ defines a line of singular models separately discussed in Eqs. (3.29)-(3.30). These are not physical singularities but rather singularities of the parametrization. ${ }^{11}$

Always in Fig. 2 the lines $F_{E}=1 / 2$ and $F_{B}=-1 / 2$ are the zeroes of $\sigma$ and of $1-\sigma$. Both models are regular; the corresponding spectra have logarithmic corrections either in the electric or in the magnetic case. For the sake of simplicity, we shall exclude these three lines when charting the area of the parameter space in the plane $\left(F_{B}, F_{E}\right)$; this is not crucial since we are just excising three lines from a whole plane. Furthermore these cases have been separately discussed in the previous sections; they are not more significant than the others and they are also irrelevant for the phenomenological considerations discussed in Sec. V.

\footnotetext{
${ }^{11}$ As previously shown in this case the susceptibilities have an exponential dependence on $\eta$ and, consequently, the relation between $\tau$ and $\eta$ is logarithmic. These models are characterized by an electric field that vanishes exactly but besides this they do not have a particularly relevant status from the viewpoint of phenomenology.
}

TABLE I. First quadrant $F_{E}>0$ and $F_{B}>0$.

\begin{tabular}{lcc}
\hline \hline $\begin{array}{l}\text { Normalized } \\
\text { rates }\end{array}$ & \multicolumn{1}{c}{$n_{B}$} & $n_{E}$ \\
\hline $0<F_{E}<1 / 2$ & $n_{B}=\left(5+6 F_{B}-4 F_{E}\right) /\left(1+F_{B}-F_{E}\right)$ & $n_{E}=10-n_{B}$ \\
$1 / 2<F_{E}$ & $n_{B}=\left(7+6 F_{B}-8 F_{E}\right) /\left(1+F_{B}-F_{E}\right)$ & $n_{E}=n_{B}-2$ \\
$\quad<F_{B}+1$ & & \\
$F_{E}>F_{B}+1$ & $n_{B}=\left(5+6 F_{B}-4 F_{E}\right) /\left(1+F_{B}-F_{E}\right)$ & $n_{E}=n_{B}+2$ \\
\hline \hline
\end{tabular}

TABLE II. Third quadrant $F_{E}<0$ and $F_{B}<0$.

\begin{tabular}{lll}
\hline \hline $\begin{array}{l}\text { Normalized } \\
\text { rates }\end{array}$ & \multicolumn{1}{c}{$n_{B}$} & $n_{E}$ \\
\hline $0<F_{B}<1 / 2$ & $n_{B}=10-n_{E}$ & $n_{E}=\left(5-6 F_{E}+4 F_{B}\right) /\left(1+F_{B}-F_{E}\right)$ \\
$1 / 2<F_{E}$ & $n_{B}=2+n_{E}$ & $n_{E}=\left(7+8 F_{B}-6 F_{E}\right) /\left(1+F_{B}-F_{E}\right)$ \\
$<F_{B}+1$ & & \\
$F_{E}>F_{B}+1$ & $n_{B}=2-n_{E}$ & $n_{E}=\left(5-6 F_{E}+4 F_{B}\right) /\left(1+F_{B}-F_{E}\right)$ \\
\hline
\end{tabular}

In Fig. 2 there are finally two particularly simple cases, namely $F_{E}=0$ (with $F_{B}$ free to vary) and $F_{B}=0$ (with $F_{E}$ free to vary). These two situations correspond to the situation where one of the gauge couplings is constant.

Different portions of Figs. 1 and 2 can be easily related via duality. Equation (4.11) refers to the first quadrant of Figs. 1 and 2. Let us now suppose to be interested in the electric and magnetic spectral indices in the third quadrant where $F_{E}<0$ and $F_{B}<0$. In this region both gauge couplings are decreasing. The three regions of Eq. (4.11) transform under duality into

$$
\begin{aligned}
-1 / 2<F_{B}<0, & 0<\sigma<1, \\
-1+F_{E}<F_{B}<-1 / 2, & \sigma>1, \\
F_{E}>F_{B}+1, & \sigma<0 .
\end{aligned}
$$

The result of Eq. (4.12) can be easily derived by recalling that, under duality, $F_{E} \rightarrow-F_{B}$ and $F_{B} \rightarrow-F_{E}$. Notice also that duality relates the first and third quadrant (region by region) given the transformation properties of $\sigma$. The results of the duality transformation are reported in Table II. In the remaining two quadrants the same logic applies so that the spectra, the spectral indices, and the other information can be easily deduced by successive duality transformations from the first or from the third quadrants. ${ }^{12}$

Before discussing in detail the parameter space of the model, let us remark that there are, broadly speaking, three distinct physical situations. The gauge couplings may be both small at $a_{i}$ and then increase: this is the case of weakly coupled initial conditions evolving towards strong coupling at the end of inflation. The second possibility is that the

\footnotetext{
${ }^{12}$ Notice that from Table I the structure of Table II can be immediately obtained by only using duality.
} 
gauge couplings are initially of order 1 and then decrease towards the end of inflation: this is the case of the strongly coupled initial conditions. The third possibility is that initially one of the gauge couplings is strong while the other is weak; this is the case of mixed initial conditions. In the case of conventional inflationary models, the Universe evolves from strong gravitational coupling to weak gravitational coupling, i.e., the space-time curvature is maximal at the onset of inflation and gets smaller during reheating. It is fair to say that the potential drawbacks of magnetogenesis coincide with the potential drawbacks of conventional models of inflation which are, typically, not geodesically complete in their past history and preceded by a high curvature regime. The considerations reported here can be easily extended to the case of bouncing models evolving from weak gravitational coupling to strong gravitational coupling, i.e., the space-time curvature is small initially and gets larger at the reheating.

In summary, it would be pedantic to discuss in details all the six regions of the parameter space of Fig. 1 (or Fig. 2). So it is mandatory to complement Figs. 1 and 2 with more physical considerations. Fortunately duality comes to our rescue since the different portions of the parameter space are all related by duality transformations so that, for a complete discussion of the parameter space, a thorough examination of a single quadrant will suffice. Since the possibility of having the simultaneous growth of the gauge couplings during inflation is typical of this type of model, we shall therefore focus on the first quadrant where $F_{E}>0$ and $F_{B}>0$.

\section{CHARTING THE PARAMETER SPACE OF MAGNETOGENESIS}

The parameter space of inflationary magnetogenesis will now be charted by discussing in detail the first quadrant of Figs. 1 and 2. Thanks to the duality symmetry the remaining portions of the parameter space will be easy to discuss (see Sec. IV for a specific discussion of this point). Three independent requirements are relevant for the present discussion: the critical density bound (sometimes dubbed backreaction constraint), the magnetogenesis requirements and the naturalness of the initial conditions. All the mentioned conditions are necessary but none of them is per se sufficient to pin down the allowed region of the $\left(F_{B}, F_{E}\right)$ plane. However, if they are all jointly verified in a given domain they become sufficient.

The logic will be, in short, the following. We shall first identify and classify all the domains of the $\left(F_{B}, F_{E}\right)$ plane where the backreaction constraints are satisfied. Then, in the regions not constrained by backreaction we shall compute precisely the magnetic power spectrum by keeping track of all terms and prefactors of the spectra. It will then be easier to see in the $\left(F_{E}, F_{B}\right)$ plane where and how the weakly coupled initial conditions are verified.

\section{A. Maximal wavenumber of the spectra}

We recall that $k \tau$ can be expressed as

$$
k \tau=\frac{k}{(1-\epsilon) a H}=\frac{k}{H_{0}} e^{-N_{\max }}\left[1+\epsilon+\mathcal{O}\left(\epsilon^{2}\right)\right],
$$

where $H_{0}=2.334 \times 10^{-4}\left(h_{0} / 0.7\right) \mathrm{Mpc}^{-1}$ is the present value of the Hubble rate and $N_{\max }$ is the maximal number of efolds which are today accessible to our observations [25] (see also [26]). In practice $N_{\max }$ is determined by fitting the redshifted inflationary event horizon inside the present Hubble radius $H_{0}^{-1}$ :

$$
e^{N_{\max }}=\left(2 \pi \epsilon \mathcal{A}_{\mathcal{R}} \Omega_{\mathrm{R} 0}\right)^{1 / 4}\left(\frac{M_{\mathrm{P}}}{H_{0}}\right)^{1 / 2}\left(\frac{H_{r}}{H}\right)^{\gamma-1 / 2},
$$

where $\Omega_{\mathrm{R} 0}$ is the present critical fraction of radiation (in the concordance model $h_{0}^{2} \Omega_{\mathrm{R} 0}=4.15 \times 10^{-5}$ ). The term containing $\gamma$ accounts for the possibility of a delayed reheating ending at a putative scale $H_{r}$ eventually much smaller than the inflationary curvature scale $H$. For illustration we shall focus on the simplest case and choose the sudden reheating approximation by setting $\gamma=1 / 2$. Different possibilities can be however considered. A long postinflationary phase dominated by a stiff equation of state has been examined in the context of magnetogenesis (see first paper of Ref. [25] and references therein). A delayed reheating has the effect of increasing $N_{\max }$. The largest value of $N_{\max }$ in the case of a stiff postinflationary phase can be estimated as $N_{\max }=78.3+(1 / 3) \ln \epsilon$. In the sudden reheating approximation we have $N_{\max } \simeq 63.25+0.25 \ln \epsilon$ which is numerically close to the minimal number of efolds $N_{\min }$ needed to solve the kinematic problems of the standard cosmological model (i.e., $N_{\min } \simeq N_{\max }$ ). Recall, as usual, that $\mathcal{A}_{\mathcal{R}}$ denotes the fiducial amplitude of the scalar power spectrum at the pivot scale $k_{p}=0.002 \mathrm{Mpc}^{-1}$. We shall not deviate from these fiducial values and assume the standard concordance lore for all the late time cosmological parameters.

In Fig. 3 we present the contours of constant $\log \Omega_{E}$ (the common logarithm critical fraction of electric energy density) and the contours of constant $\log \Omega_{B}$ (the common logarithm of the critical fraction of the magnetic energy density). In Fig. 3 the spectra have been evaluated at the maximal wavenumber $k \tau=1$. This is strictly speaking not the highest frequency of the electric power spectrum which is exponentially suppressed as soon as the reheating starts. ${ }^{13}$ To extend artificially the electric power spectrum beyond the frequency fixed by the postinflationary conductivity (for more details see $[22,25]$ ) implies that the resulting constraints are much stronger. Since we are here

\footnotetext{
${ }^{13}$ Imposing the correct boundary conditions between the inflationary phase and the postinflationary phase entails also an overall suppression of the electric power spectrum in comparison with the magnetic power spectrum [25].
} 

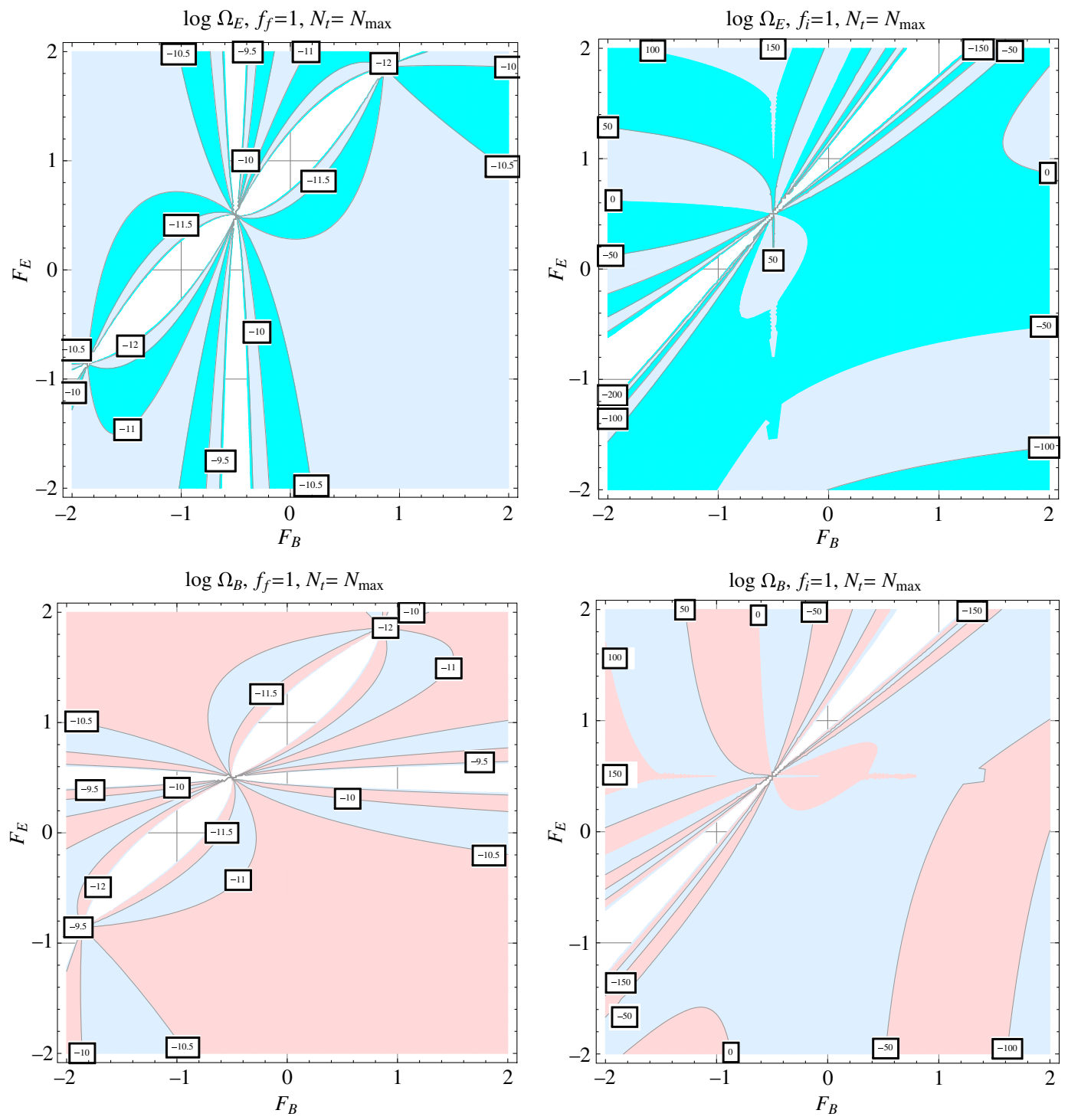

FIG. 3 (color online). The contours for constant $\log \Omega_{B}$ and for constant $\log \Omega_{E}$. The plots on the left refer to the case $f_{f}=1$ and for $N_{t}=N_{\max }=62.09$; the plots on the right refer to the case $f_{i}=1$. The various labels illustrate the values of the critical fractions on the given curve. On the horizontal axis we illustrate the rate of variation of $g_{B}$ while on the vertical axis the rate of variation of $g_{E}$ is illustrated.

trying to constrain the scenario it makes sense to impose here the most demanding requirements at the most demanding scale.

In Fig. 3 the values of the rates encompass all the four quadrants of Figs. 1 and 2. This choice has been made also for illustrative reasons since, as explained above, in the remaining figures of the section we shall limit our attention to the first quadrant. The results of Fig. 3 illustrate the domains of the parameter space where the corresponding critical fractions are still perturbative around the maximal wavenumber of the spectra. The contours where one of the two critical fractions is larger than 1 should be ideally excised from the physical region of the parameter space. We note that the classes of models with $f_{f}=1$ seem to be comparatively less constrained than the ones with $f_{i}=1$. In Fig. $3 F_{E}=F_{B}+1$ and $F_{E}=1 / 2$ have been excised. ${ }^{14}$ In Fig. 3 and in the forthcoming figures we have chosen, as already mentioned, $\mathcal{A}_{\mathcal{R}}=2.41 \times 10^{-9}$ and $\epsilon=0.01$.

The results of Fig. 3 also fix the allowed excursion of $F_{E}$ and $F_{B}$. Indeed at the maximal frequency of the spectrum the amplitude depends also on the maximal value of $F_{E}$ and $F_{B}$. In Fig. 3, for illustrative reasons, we used values $\left|F_{B}\right|<$ 2 and $\left|F_{E}\right|<2$. Each value of $f_{i}$ (or $\left.f_{f}\right)$ is compatible with a maximal range of $F_{E}$ and $F_{B}$. For instance, if $f_{f}=\mathcal{O}(1)$ we have that, at most $\left|F_{B}\right|<5$ and $\left|F_{E}\right|<5$ : larger values

\footnotetext{
${ }^{14}$ As discussed in Eqs. (3.29) and (3.30) this is due to a singularity of the parametrization (not of the model).
} 
imply that $\Omega_{E} \simeq \mathcal{O}(1)$ around the maximal wavenumber of the spectrum.

\section{B. Backreaction constraints}

If the total energy density of the large-scale modes does not exceed the critical energy density, the following integral

$$
\begin{aligned}
& \mathcal{F}\left(f_{i}, \sigma, \mu\right) \int_{k_{i} \tau}^{k_{f} \tau} \frac{d x}{x}\left[\mathcal{Q}_{B}(\sigma, \mu) x^{5-2|\sigma|}+\mathcal{Q}_{E}(\sigma, \mu) x^{5-2|\sigma-1|}\right], \\
& \mathcal{F}\left(f_{i}, \sigma, \mu\right)=\frac{2 \pi}{3} \epsilon \mathcal{A}_{\mathcal{R}} f_{i}^{|\sigma|-1}\left(\frac{a}{a_{i}}\right)^{2 \mu(|\sigma|-1)}
\end{aligned}
$$

cannot exceed 1. In practice it will also have to be much smaller than 1, i.e., at most $10^{-3}$. In the most constraining situation the integral goes from $k_{i}=1 / \tau_{i}$ (corresponding to the mode leaving the Hubble radius when $a=a_{i}$ ) up to $k_{f}=1 / \tau_{f}$ (corresponding to the mode reentering the Hubble radius when at $a=a_{f}$ ). Performing explicitly the integrals in the case $2|\sigma| \neq 5$ and $2|\sigma-1| \neq 5$, Eq. (5.3) implies ${ }^{15}$

$$
\begin{gathered}
\mathcal{F}\left(f_{i}, \sigma, \mu\right)\left\{\frac{\mathcal{Q}_{B}(\sigma, \mu) x_{i}^{5-2|\sigma|}}{5-2|\sigma|}\left[\left(\frac{a_{i}}{a_{f}}\right)^{5-2|\sigma|}-1\right]\right. \\
\left.+\frac{\mathcal{Q}_{E}(\sigma, \mu) x_{i}^{5-2|\sigma-1|}}{5-2|\sigma-1|}\left[\left(\frac{a_{i}}{a_{f}}\right)^{5-2|\sigma-1|}-1\right]\right\} .
\end{gathered}
$$

To analyze the requirements imposed by Eq. (5.4) we shall first consider the limit $\mathcal{Q}_{E} \rightarrow 1$ and $\mathcal{Q}_{B} \rightarrow 1$. With this approximation it will be easier to pin down the area of the allowed region in the $\left(F_{B}, F_{E}\right)$ plane. This preliminary step will then be supplemented by the accurate contour plots obtained with the exact form of $\mathcal{Q}_{E}$ and $\mathcal{Q}_{B}$. Indeed the corrections due to $\mathcal{Q}_{E}$ and $\mathcal{Q}_{B}$ can be neglected in the first approximation but they are not irrelevant.

Thus, in the limit $\mathcal{Q}_{E} \rightarrow 1$ and $\mathcal{Q}_{B} \rightarrow 1$, Eq. (5.4) implies that the following three inequalities must be separately satisfied in the physical region of the parameter space:

\footnotetext{
${ }^{15}$ The cases $2|\sigma|=5$ and $2|\sigma-1|=5$ must be separately treated since the integral lead to logarithmic divergences. More specifically, if $\sigma=5 / 2$ and $f_{f}=1$, then everything is okay besides the logarithmic growth which is not essential for the bound. Conversely, if $\sigma=5 / 2$ and $f_{i}=1$ we must anyway demand $\mu<0$. If $\sigma=-5 / 2$ and $f_{f}=1$ the spectra are excluded since they get easily overcritical. In the case $\sigma=-5 / 2$ and $f_{i}=$ 1 the spectra are viable provided $3 \mu+2<0$. When $\sigma=7 / 2$ and $f_{f}=1$ the spectra are excluded while for $f_{i}=1$ they are only compatible provided $5 \mu+2<0$. Finally, if $\sigma=-3 / 2$ the constraints are under control for $f_{f}=1$ (and for $f_{i}=1$ but provided $\mu<0$ ).
}

$$
\begin{aligned}
(|\sigma|-1)\left[\frac{\ln f_{i}}{N_{t}}+2 \mu\right] & \leq 0, \\
(|\sigma|-1)\left[\frac{\ln f_{i}}{N_{t}}+2 \mu\right]-5+2|\sigma| & \leq 0, \\
(|\sigma|-1)\left[\frac{\ln f_{i}}{N_{t}}+2 \mu\right]-5+2|\sigma-1| & \leq 0,
\end{aligned}
$$

where $N_{t}$ denotes, as usual, the total number of efolds. Recalling that $\ln \left(f_{f} / f_{i}\right)=2\left(F_{B}-F_{E}\right) N_{t}$, the two complementary physical situations correspond to $f_{f}=1$ and to $f_{i}=1$. Indeed these two cases well represent the effect of the initial conditions on the allowed region of the parameter space. If $f_{i} \neq 1$ [but $f_{i} \simeq \mathcal{O}(1)$ ] the situation is qualitatively close to the case $f_{i}=1$; if $f_{i} \gg 1$ the qualitative features of the spectra are close to the case $f_{f}=1$.

\section{The case $f_{f}=1$}

In this case he backreaction constraints can be evaded provided

$$
5-2|\sigma| \geq 0, \quad 5-2|\sigma-1| \geq 0 .
$$

When $\sigma>1$ the conditions imposed by Eq. (5.6) imply, respectively, $\sigma \leq 5 / 2$ and $\sigma \leq 7 / 2$. Thus, in the domain $\sigma>1$ the most constraining bound is $\sigma \leq 5 / 2$. In the region $0<\sigma<1$ Eq. (5.6) demands that $-3 / 2 \leq \sigma \leq 5 / 2$. But both conditions are always verified in the interval $0<\sigma<1$. Therefore no supplementary bounds arise in this case. Finally, if $\sigma<0$ Eq. (5.6) demands $\sigma \geq-5 / 2$ and $\sigma \geq-3 / 2$. The most constraining of the two previous conditions is $\sigma \geq-3 / 2$. In Fig. 2 the three domains $\sigma>1,0<\sigma<1$ and $\sigma<0$ have been specifically illustrated in the $\left(F_{B}, F_{E}\right)$ plane.

Collecting together all the relevant requirements, in the case $f_{f}=1$ the bounds of Eqs. (5.3) and (5.4) imply that $-3 / 2 \leq \sigma \leq 5 / 2$. Recalling Eq. (4.2) we then have the following requirement in the $\left(F_{B}, F_{E}\right)$ plane:

$$
-3<\frac{1-2 F_{E}}{1+F_{B}-F_{E}}<5 .
$$

The inequalities of Eq. (5.7) are verified in the following two nonoverlapping regions of the first quadrant (i.e., $F_{E} \geq$ 0 and $\left.F_{B} \geq 0\right)$ of the $\left(F_{B}, F_{E}\right)$ plane:

$$
\begin{array}{ll}
F_{E}>F_{B}+1, & F_{E} \geq \frac{5}{3} F_{B}+\frac{4}{3}, \\
F_{E}<1+F_{B}, & F_{E} \leq \frac{3}{5} F_{B}+\frac{4}{5} .
\end{array}
$$

Notice that in the region of Eq. (5.8) the electric coupling grows always faster than the magnetic coupling (i.e., $F_{E}>F_{B}$ ) and consequently $f$ decreases (i.e., $F<0$ ). 

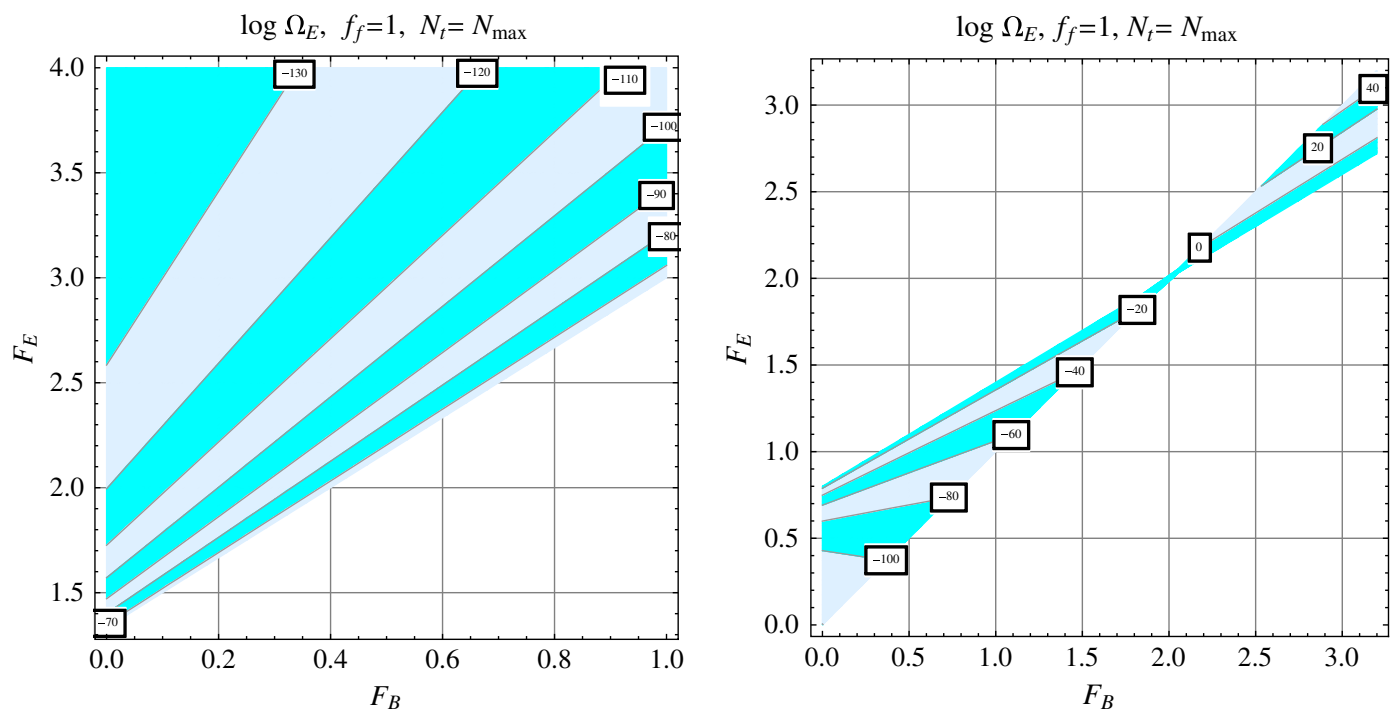

FIG. 4 (color online). The two contour plots illustrate the common logarithm of $\Omega_{E}$ without any simplifying approximation. In the left plot the region of Eq. (5.8) is illustrated. The plot on the right refers instead to the region of Eq. (5.9). In both cases the wavenumber has been chosen to be $10^{-4} \mathrm{Mpc}^{-1}$.

Conversely in the region determined by Eq. (5.9) we have that $F_{E} \geq F_{B}$ when $0<F_{B} \leq 2$ and $F_{E}<F_{B}$ whenever $F_{B}>2$. The straight line $F_{E}=5 F_{B} / 3+4 / 3$ corresponds to the case of a flat magnetic power spectrum (i.e., $n_{B} \rightarrow 1$ ). This is an isospectral line in the sense that along this line the magnetic and the electric spectral indices do not change. Conversely the amplitude of the spectrum still has some dependence on $F_{B}$.

The results of Eqs. (5.8) and (5.9) are confirmed by the accurate analysis of the relevant contour plots. In the left and right plots of Fig. 4 we illustrate the common logarithm of $\Omega_{E}$ in the case of the regions discussed, respectively, in Eqs. (5.8) and (5.9). The power spectra are evaluated for wavenumbers comparable with the infrared branch of the spectrum, i.e., typically of the order of $10^{-4} \mathrm{Mpc}^{-1}$. Larger wavenumbers relevant for the magnetogenesis problem [i.e., $k=\mathcal{O}\left(\mathrm{Mpc}^{-1}\right)$ ] lead to slightly larger figures which are however always of the same order. This is due to the quasiflatness of the spectra in this domain. At the highest frequency the plots of Fig. 3 apply and, even there, the energy densities are much smaller than the critical one. In both plots $N_{t}=N_{\max }=62.09$; the various labels illustrate the values of the critical fraction on the given curve. The shaded areas illustrates the regions where, according to Eqs. (5.8) and (5.9) the backreaction constraints are satisfied.
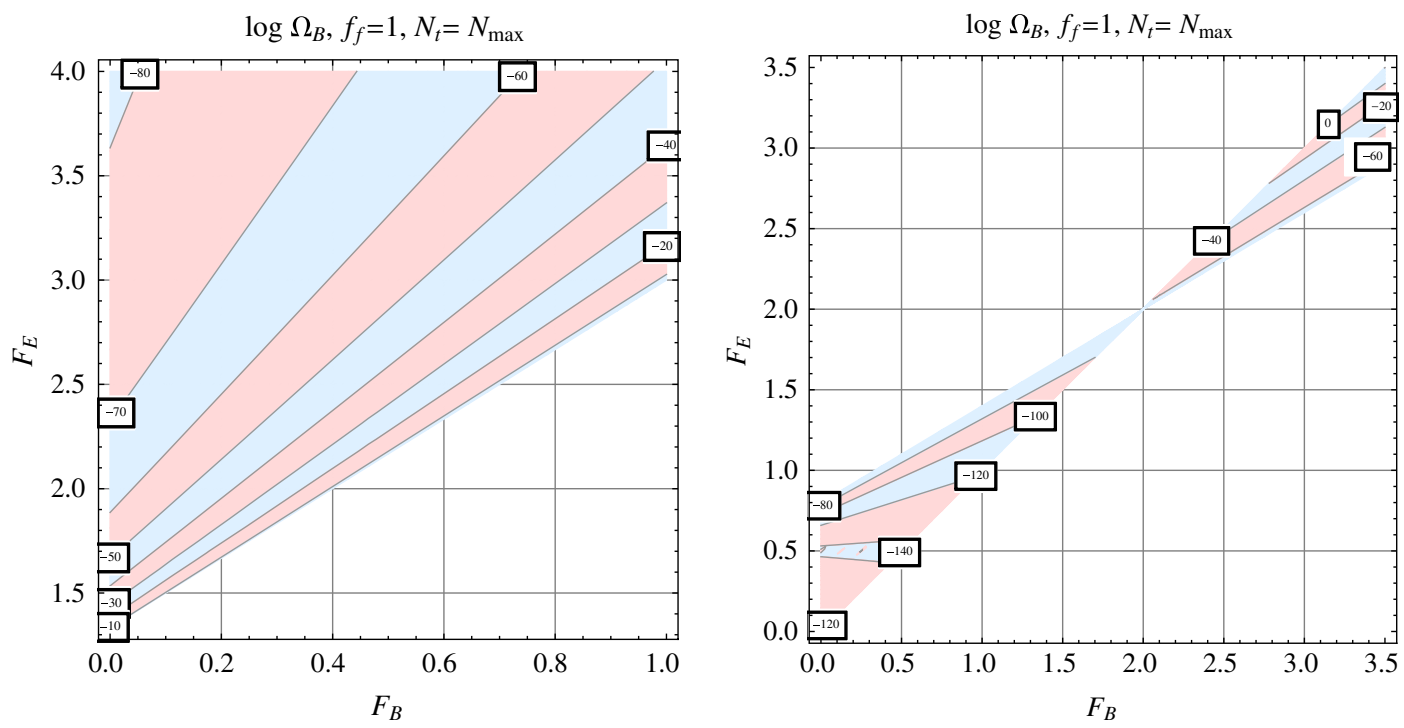

FIG. 5 (color online). The contours of constant $\log \Omega_{B}$ in the case of Eq. (5.8) (plot at the right) and in the case of Eq. (5.9) (plot at the left); the typical scale has been chosen, as in Fig. 4, to coincide with $k=1 \mathrm{Mpc}^{-1}$. 
In Fig. 5 we illustrate the same cases discussed in Fig. 4 but in terms of the common logarithm of $\Omega_{B}$. The fiducial scale is always $k=10^{-4} \mathrm{Mpc}^{-1}$ and the other parameters are also unchanged in comparison with Fig. 4. The combination of Figs. 3, 4 and 5 shows that the backreaction constraints are satisfied both for the electric and for the magnetic fields in the physical range of wavenumbers. In the last part of this section the safe regions pinned down by the backreaction constraints will be confronted with the magnetogenesis requirements.

\section{The case $f_{i}=1$}

We are now going to address the complementary case where $f_{i}=1$. According to Eq. (5.5) in the case $f_{i}=1$ the following inequalities must be satisfied:

$$
\begin{aligned}
& -2 \mu(|\sigma|-1)+5-2|\sigma| \geq 0, \\
& -2 \mu(|\sigma|-1)+5-2|\sigma-1| \geq 0, \\
& -2 \mu(|\sigma|-1) \geq 0 .
\end{aligned}
$$

If $\sigma>1$, Eq. (5.10) implies that the two most constraining inequalities are $2 \mu(\sigma-1) \leq 0$ and $5-2 \sigma \geq 2 \mu(\sigma-1)$. In the plane $\left(F_{B}, F_{E}\right)$ this requirement translates into the following triplet of inequalities:

$$
F_{E}>F_{B}+1, \quad F_{E}<F_{B}, \quad F_{B} \geq-2
$$

notice that $F_{B} \geq-2$ is automatically satisfied since we work in the region $F_{B} \geq 0$ and $F_{E} \geq 0$.

If $\sigma<0$ the conditions of Eq. (5.10) become

$$
\begin{gathered}
2 \mu(\sigma+1) \geq 0, \quad 5+2 \sigma \geq-2 \mu(\sigma+1), \\
5+2 \sigma \geq 2-2 \mu(\sigma+1) .
\end{gathered}
$$

The last inequality of Eq. (5.12) is the most constraining so that $-3 / 2-\mu(\sigma+1) \leq \sigma<0$. In terms of $F_{E}$ and $F_{B}$ this range of $\sigma$ translates into:

$$
\begin{gathered}
\frac{1-2 F_{E}}{2\left(1+F_{B}-F_{E}\right)} \leq 0, \quad \frac{\left(F_{B}-F_{E}\right)\left(3+2 F_{B}-4 F_{E}\right)}{1+F_{B}-F_{E}} \geq 0, \\
F_{E} \leq 1+\frac{F_{B}}{2} .
\end{gathered}
$$

If $1+F_{B}-F_{E}>0$, then $F_{E}>1 / 2$ and the inequalities defining the allowed regions are ${ }^{16}$

$$
\left(F_{B}-F_{E}\right)\left(3+2 F_{B}-4 F_{E}\right) \geq 0, \quad F_{E}<1+F_{B} / 2 .
$$

\footnotetext{
${ }^{16}$ If $1-F_{E}+F_{B}<0$, Eq. (5.13) demands that $F_{E}<1 / 2$ which is not compatible with $F_{E}>F_{B}+1$. So this possibility must be discarded.
}

For $F_{B}>F_{E}$ the allowed region is

$$
\begin{aligned}
& 1 / 2<F_{B} \leq 3 / 2, \quad 1 / 2<F_{E}<F_{B}, \\
& F_{B} \geq 3 / 2, \quad 3 / 2 \leq F_{E} \leq 3 / 4+F_{B} / 2 .
\end{aligned}
$$

For $F_{B}<F_{E}$ the allowed region is

$0 \leq F_{B} \leq 3 / 2, \quad 3 / 4+F_{B} / 2 \leq F_{E} \leq 1+F_{B} / 2$.

Finally in the region $0<\sigma<1$ the backreaction constraints are satisfied in the region $F_{E}<1 / 2$.

In Figs. 6 and 7 we illustrate, respectively, the common logarithm of $\Omega_{E}$ and of $\Omega_{B}$. The contour plots on the left refer to the region of Eq. (5.15) while the contour plots on the right illustrate the parameter range of Eq. (5.16). The fiducial set of cosmological parameters is the same as in the previous figures.

\section{Dualizing the contour plots}

The region of the parameter space explored so far coincides with the first quadrant of the $\left(F_{B}, F_{E}\right)$ plane. Using duality the contour plots of the other portions of the parameter space can be easily obtained and studied, as specifically discussed in Sec. IV. Let us just give an example of this strategy. Let us suppose, for instance, to be interested in the case where both rates are negatives, namely $F_{B}<0$ and $F_{E}<0$, as it happens in the third quadrant of Figs. 1 and 2.

Recalling therefore Eqs. (4.11) and (4.12) (and also Tables I and II) we can easily dualize all the contour plots obtained so far in the regions where the backreaction constraints are safely satisfied. In Figs. 8 and 9 we illustrate the contours plots dual to the ones reported in Figs. 4 and 5. Since, under duality, $F_{B} \rightarrow-F_{E}$ and $F_{E} \rightarrow-F_{B}$ the axes of Figs. 8 and 9 are interchanged in comparison with the axes of Figs. 4 and 5. The same exercise discussed in the case $f_{f}=1$ can be also carried on in all the other relevant cases of phenomenological interest and also in the remaining quadrants of the parameter space.

The plots reported in Figs. 8 and 9 can be related, by symmetry, to the plots of Figs. 4 and 5. Of course also the domains of the spectrum must be dualized as specifically discussed in Sec. IV. It is however appropriate to stress that Figs. 8 and 9 have been obtained by plotting explicitly the relevant critical fractions in the dual regions of Figs. 4 and 5. The obtained results show indeed that, a posteriori, duality can be directly applied also to the exclusion plots without plotting explicitly the relevant spectra.

\section{Magnetogenesis requirements}

Having determined the regions of the parameter space where the backreaction constraints are enforced we can address the problem of the magnetogenesis constraints. Let 

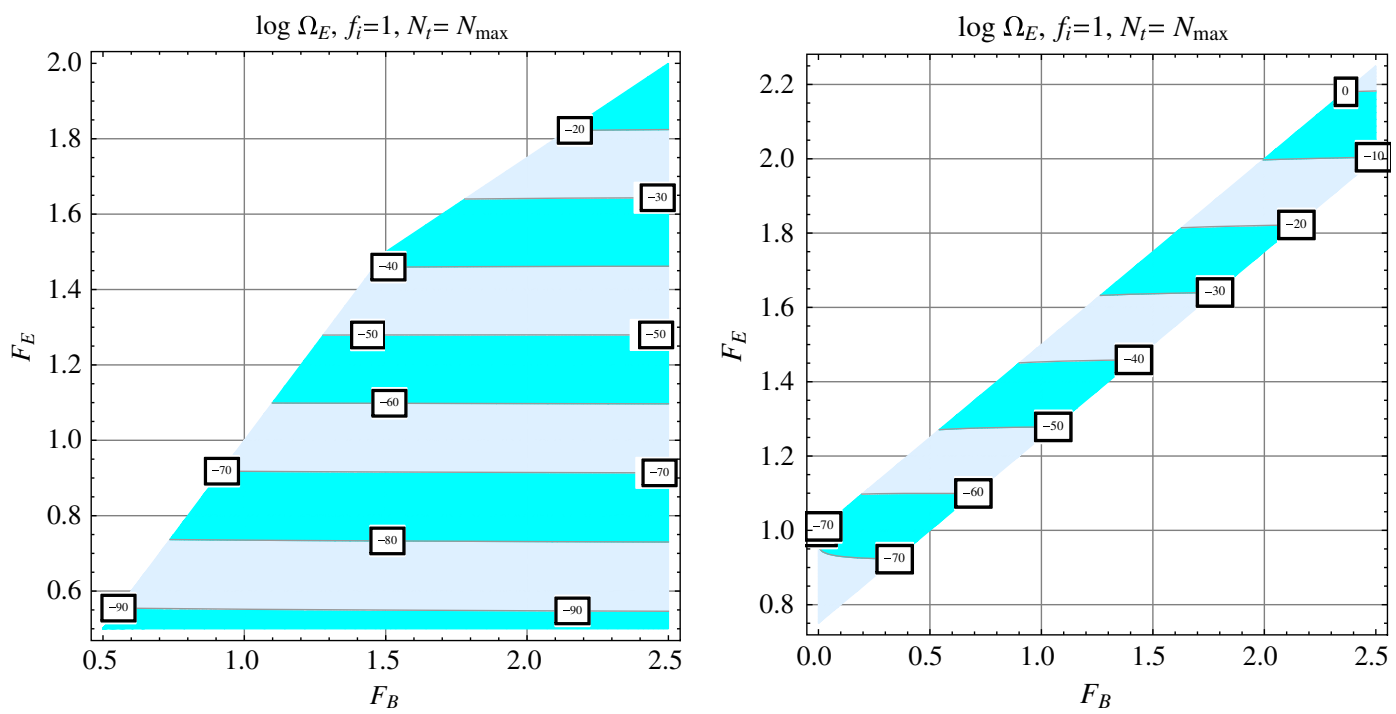

FIG. 6 (color online). The common logarithm of $\Omega_{E}$ in the region (5.15) (plot on the left) and in the region (5.16) (plot on the right). The same parameters and the same scales of Figs. 4 and 5 have been employed.
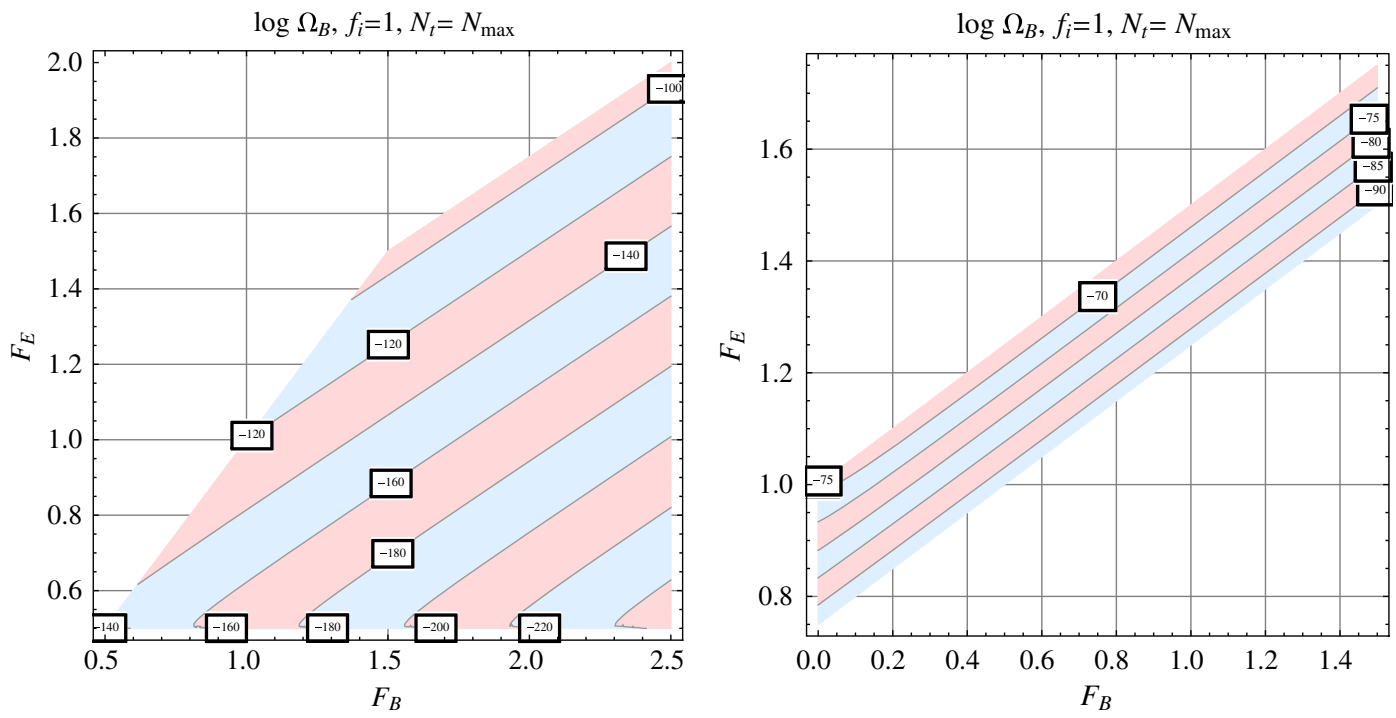

FIG. 7 (color online). The common logarithm of $\Omega_{B}$ in the region (5.15) (plot on the left) and in the region (5.16) (plot on the right). The same parameters and the same scales of Figs. 4 and 5 have been employed.

us therefore focus our attention on the first quadrant, as in the previous part of this section. In units of $\mathrm{nG}^{2}$ the magnetic power spectrum can be written as follows:

$$
\begin{aligned}
\frac{P_{B}}{\mathrm{nG}^{2}}= & 10^{-3.05}\left(\frac{h_{0}^{2} \Omega_{R 0}}{4.15 \times 10^{-5}}\right)\left(\frac{\mathcal{A}_{\mathcal{R}}}{2.41 \times 10^{-9}}\right) \\
& \times\left(\frac{\epsilon}{0.01}\right) \mathcal{Q}_{B}\left(n_{B}, \mu\right)\left(\frac{k}{H_{0}}\right)^{n_{B}-1} \\
& \times e^{-N_{\max }\left(n_{B}-1\right)} f_{i}^{\left(4-n_{B}\right) / 2} e^{\mu N_{t}\left(4-n_{B}\right)} .
\end{aligned}
$$

The magnetogenesis requirements roughly demand that the magnetic fields at the time of the gravitational collapse of the protogalaxy should be approximately larger than a (minimal) power spectrum which can be estimated between $10^{-32} \mathrm{nG}$ and $10^{-22} \mathrm{nG}$. The most optimistic estimate is derived by assuming that every rotation of the galaxy would increase the magnetic field of one efold. The number of galactic rotations since the collapse of the protogalaxy can be estimated between 30 and 35, leading approximately to a purported growth of 13 orders of magnitude. During collapse of the protogalaxy compressional amplification will increase the field of about 5 orders of magnitude. Thus the required seed field at the onset of the gravitational collapse must be, at least, as large as $10^{-15} \mathrm{nG}$ or, more realistically, larger than $10^{-11} \mathrm{nG}$. 

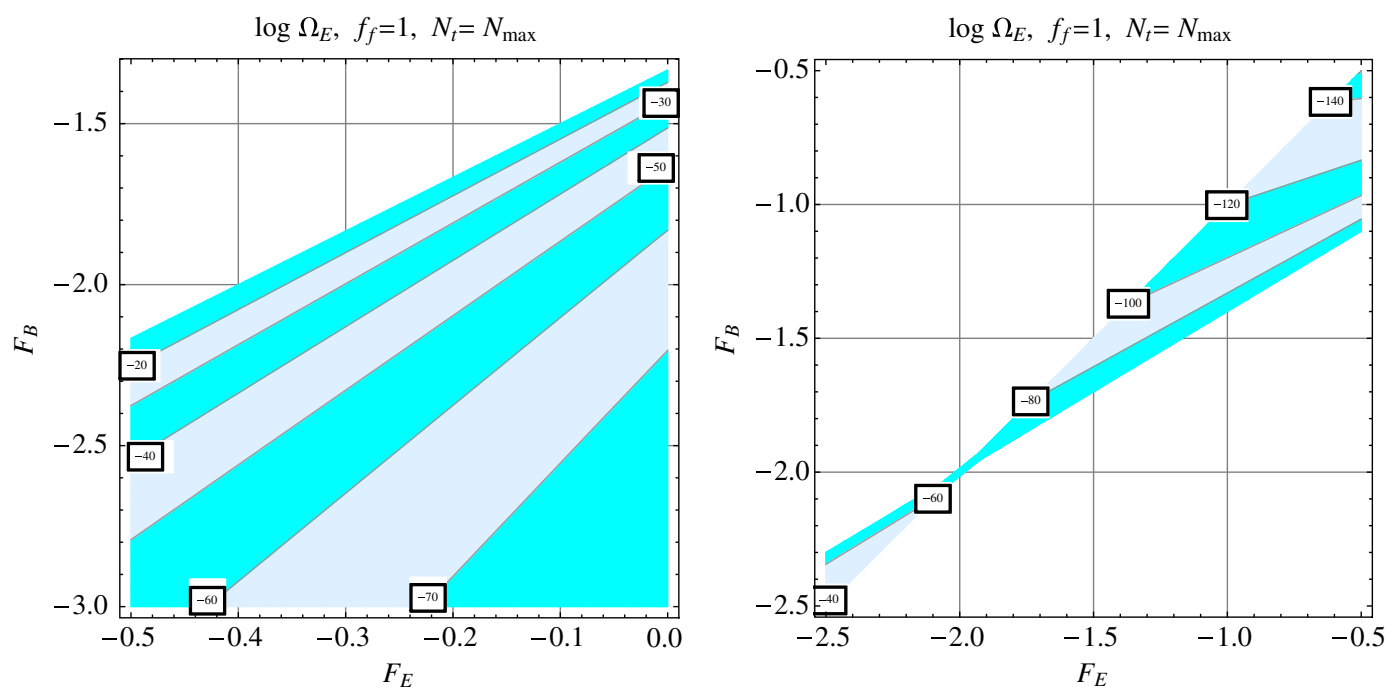

FIG. 8 (color online). We illustrate the contour plots obtained by duality from Fig. 4 . Note that the axes are interchanged thanks to duality. The notations and the parameters are the same as in Fig. 4.
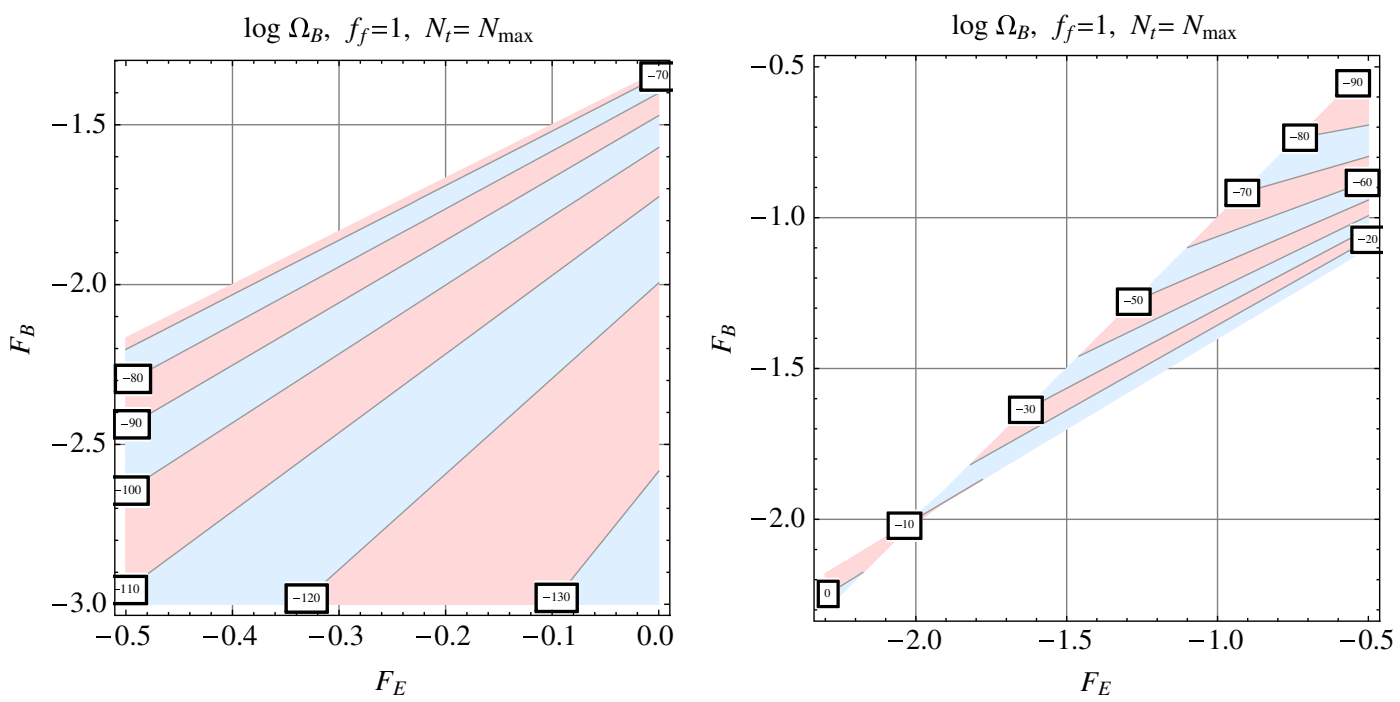

FIG. 9 (color online). We illustrate the contour plots obtained by duality from Fig. 5. Note that the axes are interchanged thanks to duality. The notations and the parameters are the same as in Fig. 5.

The simplest way to implement the magnetogenesis requirements is therefore to plot the magnetic power spectrum in units of $\mathrm{nG}^{2}$ for all the regions where the backreaction constraints are satisfied. The areas of the parameter space where

$$
\log \left(\frac{P_{B}}{\mathrm{nG}^{2}}\right) \geq-\lambda, \quad 22<\lambda<32
$$

will therefore offer viable models of magnetogenesis. Depending on the sign of the rates and on their relative magnitude, it will therefore be possible to see whether the selected models will evolve either from a strongly coupled or from a weakly coupled regime.
Let us therefore start with the case where $f_{f}=1$. The relevant regions of the parameter space have been already discussed in Figs. 4 and 5. For the same regions we shall now plot the common logarithm of $P_{B} / \mathrm{nG}^{2}$ and check if and where the condition of Eq. (5.18) is satisfied. The result of this procedure is reported in Fig. 10 where the labels in the contour plot refer to the values of the power spectrum in units of $\mathrm{nG}^{2}$. The plot on the left in Fig. 10 refers to the same region discussed in the plots on the left in Figs. 4 and 5; similarly the plots on the right correspond to the plots on the right in Figs. 4 and 5. We clearly see that the requirement of Eq. (5.18) is satisfied in two distinct regions: a stripe of the left plot and a smaller stripe in the plot on the right. According to Fig. 4, however, the 

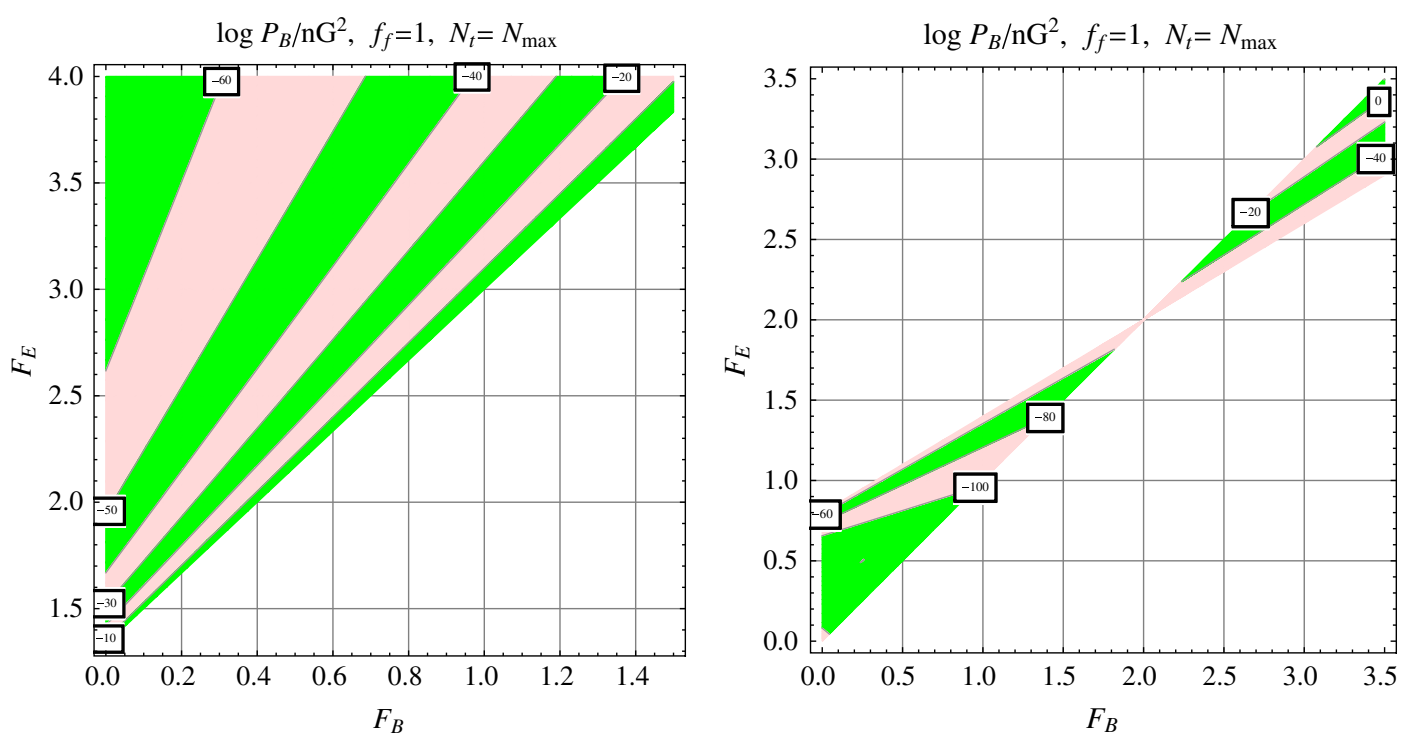

FIG. 10 (color online). We illustrate the magnetic power spectrum in units of $\mathrm{nG}^{2}$ for the regions discussed in Figs. 4 and 5 where the backreaction constraints are all satisfied.

region in the plot on the right must be excluded since in that part the electric fields are overcritical. We are therefore left with a region that can be analytically expressed as

$$
5 F_{B} / 3+4 / 3 \leq F_{E} \leq \frac{\mathcal{K}-6}{\mathcal{K}-4} F_{B}+\frac{\mathcal{K}-5}{\mathcal{K}-4}
$$

$$
\begin{aligned}
\mathcal{K} & \left(\lambda, k, N_{\max }, f_{f}\right) \\
& =\frac{3.05-\lambda+\log \left(k / H_{0}\right)-N_{\max } \log e-2 \log f_{f}}{\log \left(k / H_{0}\right)-N_{\max } \log e-0.5 \log f_{f}} .
\end{aligned}
$$

In the case of the fiducial set of parameters corresponding to the previous figures we have that the above slice becomes

$5 F_{B} / 3+4 / 3 \leq F_{E} \leq 1.46+1.91 F_{B}, \quad \lambda=22$,

$5 F_{B} / 3+4 / 3 \leq F_{E} \leq 1.56+2.13 F_{B}, \quad \lambda=32$.

The lower bound in Eq. (5.19) corresponds to the slightly distorted straight line appearing both in Figs. 4 and 10. The upper bound has been obtained from the approximate shape of the slice defined by the corresponding contour plot. In this region both gauge couplings are increasing (no strong coupling problem) and furthermore $f$ decreases from an initially large value to $f_{f}=1$.
The same analysis discussed in the case $f_{f}=1$ can now be extended to the case $f_{i}=1$ already discussed in Figs. 6 and 7. The result of this analysis is reported in Fig. 11 where we clearly see that the magnetogenesis constraints are not satisfied.

As already mentioned in the case of the backreaction constraints, the various regions of the parameter space can be easily dualized with the purpose of discussing a given region of the parameter space once the results on the first quadrant are known. An example along this line is obtained in Fig. 12 where the magnetogenesis constraints are illustrated in the case of the dual regions already studied in Figs. 8 and 9. We can therefore see that also the region of Fig. 12 leads to a viable class of magnetogenesis models where, however, the rates are negative and the gauge couplings decrease rather than increase. There are some who say that these initial data are unnatural. Some other authors claim instead that the gauge coupling should follow the gravitational coupling. In conventional inflationary models the strong coupling is at the beginning (since the singularity is at the beginning) while in bouncing models the singularity is at the end of the inflationary phase. So the most natural choice would be to ask for decreasing gauge couplings in conventional inflationary models and increasing gauge couplings in bouncing models (see, e.g., third paper in Ref. [25]). In this scenario both options can be realized in nonoverlapping domains of the parameter space.

The cases $f_{f}=1$ and $f_{i}=1$ represent in practice the two extremes of the whole range of physical models where $f_{i} \geq 1$. The cases where $f_{i}=\mathcal{O}(1)$ are very close to the patterns discussed in the $f_{i}=1$ case. Conversely when $f_{i} \gg 1$ the situation is similar to the case $f_{f}=\mathcal{O}(1)$. It is clear that by appropriately scanning the parameter space we 

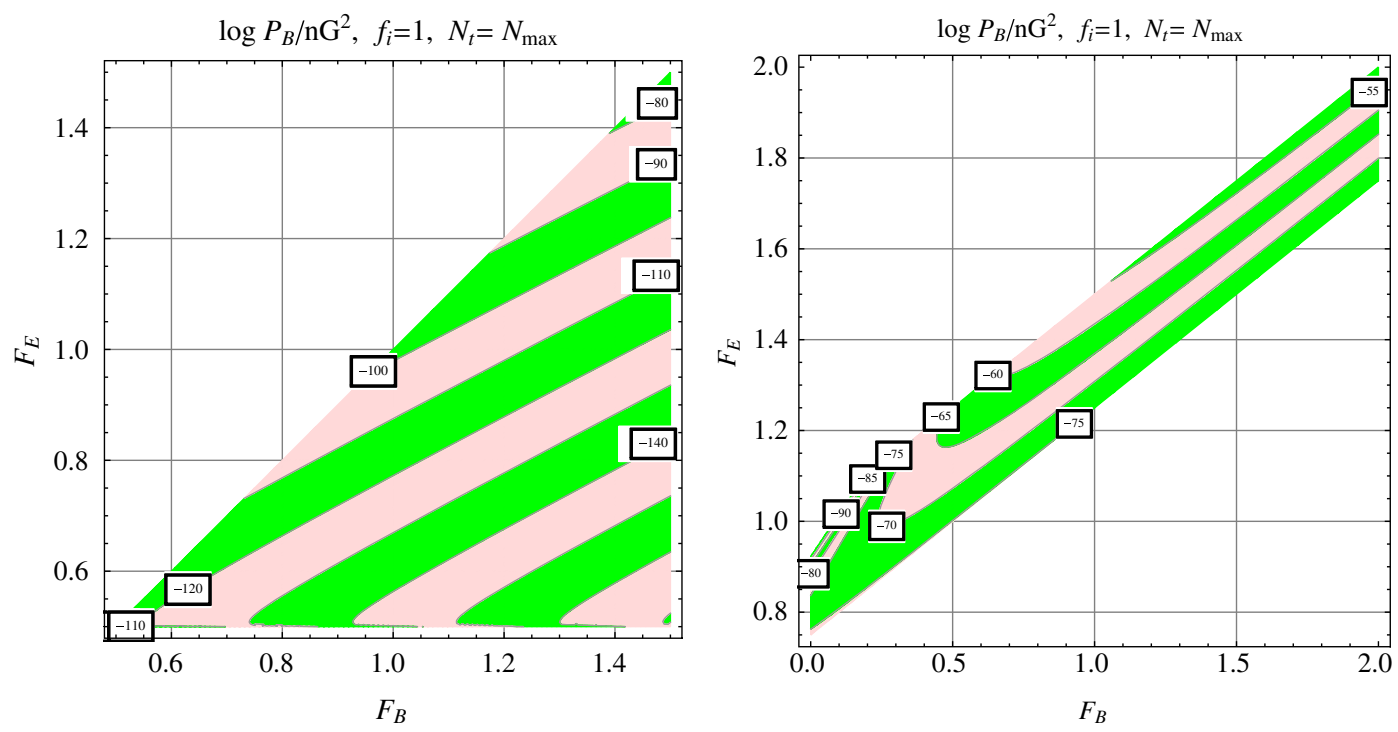

FIG. 11 (color online). We illustrate the magnetic power spectrum in units of $\mathrm{nG}^{2}$ for the regions discussed in Figs. 6 and 7 where the backreaction constraints are all satisfied.
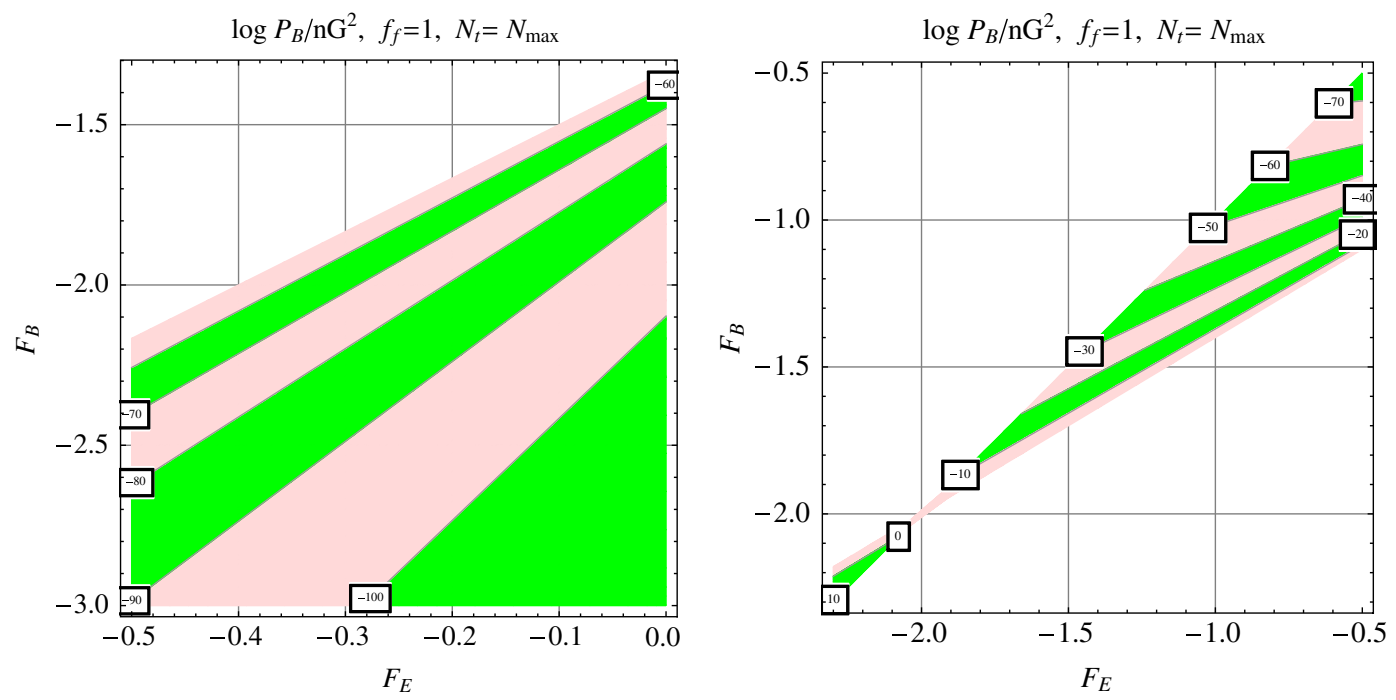

FIG. 12 (color online). We illustrate the magnetic power spectrum in units of $\mathrm{nG}^{2}$ for the regions discussed in Figs. 8 and 9 where the backreaction constraints are all satisfied.

could easily pass from an allowed region in the plane $\left(F_{B}, F_{E}\right)$ to a tuning volume in the space $\left(F_{B}, F_{E}, f_{i}\right)$. In that space the range of variation will be given exactly by the excursion discussed in the present paper, i.e., $1 \leq f_{i} \leq$ $f_{i}^{(\max )}$ where $f_{i}^{(\max )}$ coincides with $\exp \left[\left(F_{E}-F_{B}\right) N_{t}\right]$ and it corresponds to $f_{f}=1$.

Before closing this section let us also comment on the possible excursion of $N_{\max }$. As already mentioned if the reheating is delayed $N_{\max }$ can increase and get larger by even 15 efolds $[25,26]$. In this case the effect on the allowed region is minimal. For instance if $N_{\max }=78$ the area of Eq. (5.21) becomes $F_{B} / 3+4 / 3 \leq F_{E} \leq 1.42+1.84 F_{B}$ which is not different, for all practical purposes, from the previous expression.

\section{CONCLUDING REMARKS}

Magnetogenesis models based on derivative couplings have been comprehensively analyzed. A similar kind of framework arises in the relativistic generalization of Van der Waals interactions. The key aspect of this class of models is that the electric and the magnetic susceptibilities are not bound to coincide all the time, i.e., $\chi_{E} \neq \chi_{B}$. This implies that also the corresponding gauge couplings $g_{E}$ and $g_{B}$ may evolve in time at different rates. After presenting a general decomposition of the various coupling functions, the quantization of the system has been approached in terms of a newly defined time coordinate reducing to conformal time in the case when the electric and the magnetic susceptibilities coincide. 
TABLE III. Typical values of the magnetic power spectra in the allowed region and at the fiducial scale $k=1 \mathrm{Mpc}^{-1}$.

\begin{tabular}{llll}
\hline \hline$F_{B}$ & $F_{E}=5 F_{B} / 3+4 / 3$ & $F_{E}=1.46+1.91 F_{B}$ & $F_{E}=1.56+2.13 F_{B}$ \\
\hline 0 & $\sqrt{P_{B}}=10^{-2.80} \mathrm{nG}$ & $\sqrt{P_{B}}=10^{-12.25} \mathrm{nG}$ & $\sqrt{P_{B}}=10^{-16.69} \mathrm{nG}$ \\
2 & $\sqrt{P_{B}}=10^{-1.40} \mathrm{nG}$ & $\sqrt{P_{B}}=10^{-10.91} \mathrm{nG}$ & $\sqrt{P_{B}}=10^{-15.86} \mathrm{nG}$ \\
4 & $\sqrt{P_{B}}=10^{-0.89} \mathrm{nG}$ & $\sqrt{P_{B}}=10^{-10.48} \mathrm{nG}$ & $\sqrt{P_{B}}=10^{-15.52} \mathrm{nG}$ \\
\hline \hline
\end{tabular}

TABLE IV. Typical values of the magnetic spectral index in the allowed region.

\begin{tabular}{lccc}
\hline \hline$F_{B}$ & $F_{E}=5 F_{B} / 3+4 / 3$ & $F_{E}=1.46+1.91 F_{B}$ & $F_{E}=1.56+2.13 F_{B}$ \\
\hline 0 & $n_{B}=1$ & $n_{B}=1.826$ & $n_{B}=2.214$ \\
2 & $n_{B}=1$ & $n_{B}=1.807$ & $n_{B}=2.226$ \\
4 & $n_{B}=1$ & $n_{B}=1.804$ & $n_{B}=2.228$ \\
\hline \hline
\end{tabular}

It turns out that the power spectra depend on three different quantities: the normalized rates of variation of the electric and magnetic gauge couplings (i.e., $F_{E}$ and $F_{B}$ ) and also $f_{i}$, i.e., the amplitude of $g_{B}^{2} / g_{E}^{2}$ at the onset of the dynamical evolution that has been taken to coincide with the beginning of the inflationary phase. A fourth parameter measures the strength of one of the two gauge couplings at the beginning of inflation. The parameter space of the model has been scanned by using duality in order to relate the different quadrants of the $\left(F_{B}, F_{E}\right)$ plane. The physical content of the whole parameter space can therefore be reduced, via duality, to the careful analysis of the region where $F_{E}$ and $F_{B}$ are both positive semidefinite.

While the arbitrary variation of $F_{B}$ and $F_{E}$ is probably the most relevant aspect, a full account of the arbitrary variation of $f_{i}$ is not particularly significant at this stage of the analysis of the model. To explain the general trend it is sufficient to examine the case where $f_{i}=\mathcal{O}(1)$ and the case where $f_{f}=\mathcal{O}(1)$, recalling that $f_{f}$ denotes the value of $f$ at the end of inflation. The parameter space of this magnetogenesis scenario has then be accurately charted in the $\left(F_{B}, F_{E}\right)$ plane. As a consequence of this analysis the models where $f_{i}=$ $\mathcal{O}(1)$ are favored when at least one of the gauge couplings is initially strong while the models $f_{f}=\mathcal{O}(1)$ are favored when both gauge couplings are small at the beginning of inflation.

There exist wide regions in the parameter space where backreaction effects are negligible, gauge couplings are initially small and $f_{f}=\mathcal{O}(1)$. This area is defined by the corresponding excursion of the normalized rates ${ }^{17}$ in the $\left(F_{B}, F_{E}\right)$ plane:

$$
5 F_{B} / 3+4 / 3 \leq F_{E} \leq 1.46+1.91 F_{B}, \quad 0<F_{B}<5 .
$$

The functional dependence of the magnetic spectral index upon $F_{B}$ and $F_{E}$ changes from quadrant to quadrant (and

\footnotetext{
${ }^{17}$ The curves defining the allowed region depend on the total number of efolds which will be taken to coincide with $N_{\max } \simeq 63.25+0.25 \ln \epsilon$, i.e., the maximal number of efolds today accessible by observations.
}

also within the same quandrant). In the area defined by Eq. (6.1) it is given by

$$
n_{B}=\frac{5+6 F_{B}-4 F_{E}}{1+F_{B}-F_{E}}, \quad 1 \leq n_{B} \leq 1.9 .
$$

The second relation appearing in Eq. (6.2) follows from Eq. (6.1) and from the explicit expression of $n_{B}$ in the allowed region. In the range of Eqs. (6.1) and (6.2) the magnetic power spectrum at the scale of the protogalactic collapse is always larger than $10^{-22} \mathrm{nG}^{2}$. As in the case of curvature perturbations, the scale-invariant limit of the magnetic power spectrum holds for $n_{B} \rightarrow 1$ where its amplitude is

$$
\begin{aligned}
\lim _{n_{B} \rightarrow 1} \frac{P_{B}}{\mathrm{nG}^{2}}= & 10^{-2.805}\left(\frac{h_{0}^{2} \Omega_{R 0}}{4.15 \times 10^{-5}}\right)\left(\frac{\mathcal{A}_{\mathcal{R}}}{2.41 \times 10^{-9}}\right) \\
& \times\left(\frac{\epsilon}{0.01}\right)\left(\frac{1+2 F_{B}}{5}\right)^{4} .
\end{aligned}
$$

The scale-invariant amplitude still depends on $F_{B}$. If the magnetogenesis constraints are relaxed by requiring that the magnetic power spectrum at the protogalactic collapse exceeds only $10^{-32} \mathrm{nG}^{2}$ the range of allowed values of $n_{B}$ gets slightly wider, namely $1 \leq n_{B} \leq 2.2$. In Table III we illustrate the values of the magnetic power spectra in the allowed region defined by Eqs. (6.1) and (6.2). In Table IV, for the same portion of the parameter space, we report the magnetic spectral indices. Notice that the boundary lines of the allowed region are isospectral in the sense that, along these lines, the magnetic spectral index does not change or it changes very little. It is interesting to stress that, in this model, $f_{f} \rightarrow 1$ implies the equality of the gauge couplings at the end of inflation but not necessarily the coincidence of the rates. On the contrary, if the rates are coincident during inflation (i.e., $F_{E} \rightarrow F_{B}$ ) the conventional situation is recovered and the two gauge couplings evolve at the same rate. The curve $F_{E}=F_{B}$ is not isospectral and it is not included in the area defined by Eqs. (6.1) and (6.2). This occurrence shows, once more, that when $F_{E} \neq F_{B}$ the 
parameter space gets indeed wider in comparison with the conventional situation.

All in all if the magnetic and the electric susceptibilities do not coincide, the allowed regions in the parameter space of inflationary magnetogenesis gets wider in comparison with the conventional class of models. According to some, the natural initial conditions for inflationary magnetogenesis strictly demand minute gauge couplings at the beginning of inflation and larger gauge couplings at reheating. According to a different way of thinking, gauge coupling should follow the gravitational coupling: as the production of a flat spectrum of curvature perturbations demands a strong gravitational coupling in the past, similarly a quasiflat magnetic field spectrum is realized in the case of a decreasing gauge coupling which gets progressively smaller during inflation. In this scenario both options are plausible in different regions of the parameter space.
[1] H. Alfvén and C.-G. Fälthammer, Cosmical Electrodynamics, 2nd ed. (Clarendon Press, Oxford, 1963).

[2] E. N. Parker, Cosmical Magnetic Fields (Clarendon Press, Oxford, 1979).

[3] K. Enqvist, Int. J. Mod. Phys. D 07, 331 (1998); M. Giovannini, Int. J. Mod. Phys. D 13, 391 (2004); J. D. Barrow, R. Maartens, and C. G. Tsagas, Phys. Rep. 449, 131 (2007).

[4] M. Giovannini, Classical Quantum Gravity 23, R1 (2006); Phys. Rev. D 74, 063002 (2006); M. Giovannini and K. E. Kunze, Phys. Rev. D 77, 063003 (2008); 79, 103007 (2009).

[5] M. Giovannini, Phys. Rev. D 79, 121302 (2009); Classical Quantum Gravity 30, 205017 (2013).

[6] D. N. Spergel et al., Astrophys. J. Suppl. Ser. 148, 175 (2003); 170, 377 (2007); L. Page et al.,ibid. 170, 335 (2007).

[7] B. Gold et al., Astrophys. J. Suppl. Ser. 192, 15 (2011); D. Larson et al., ibid. 192, 16 (2011); C. L. Bennett et al., ibid. 192, 17 (2011); G. Hinshaw et al., ibid. 208, 19 (2013); C. L. Bennett et al., ibid. 208, 20 (2013).

[8] P. A. R. Ade et al. (Planck Collaboration), Astron. Astrophys. 571, A22 (2014); 571, A16 (2014); arXiv:1502.02114; arXiv:1502.01594; J. Chluba, D. Paoletti, F. Finelli, and J. A. Rubino-Martin, Mon. Not. R. Astron. Soc. 451, 2244 (2015).

[9] B. Ratra, Astrophys. J. Lett. 391, L1 (1992); M. Gasperini, M. Giovannini, and G. Veneziano, Phys. Rev. Lett. 75, 3796 (1995); M. Giovannini, Phys. Rev. D 56, 3198 (1997); 64, 061301 (2001).

[10] K. Bamba and M. Sasaki, J. Cosmol. Astropart. Phys. 02 (2007) 030; K. Bamba, J. Cosmol. Astropart. Phys. 10 (2007) 015; M. Giovannini, Phys. Lett. B 659, 661 (2008).

[11] K. Bamba, Phys. Rev. D 75, 083516 (2007); J. Martin and J. 'i. Yokoyama, J. Cosmol. Astropart. Phys. 01 (2008) 025; M. Giovannini, Lect. Notes Phys. 737, 863 (2008); S. Kanno, J. Soda, and M.-a. Watanabe, J. Cosmol. Astropart. Phys. 12 (2009) 009.

[12] I. A. Brown, Astrophys. J. 733, 83 (2011); N. Barnaby, R. Namba, and M. Peloso, Phys. Rev. D 85, 123523 (2012); T. Fujita and S. Mukohyama, J. Cosmol. Astropart. Phys. 10 (2012) 034; T. Kahniashvili, A. Brandenburg, L. Campanelli, B. Ratra, and A. G. Tevzadze, Phys. Rev. D 86, 103005 (2012); R. Z. Ferreira and J. Ganc, J. Cosmol. Astropart. Phys. 04 (2015) 029.

[13] R. D. Peccei and H. R. Quinn, Phys. Rev. Lett. 38, 1440 (1977); Phys. Rev. D 16, 1791 (1977).
[14] J. Kim, Phys. Rep. 150, 1 (1987); H.-Y. Cheng, ibid., 158, 1 (1988); G. G. Raffelt, Phys. Rep. 198, 1 (1990); Lect. Notes Phys. 741, 51 (2008).

[15] S. Carroll, G. Field, and R. Jackiw, Phys. Rev. D 41, 1231 (1990); W. D. Garretson, G. Field, and S. Carroll, Phys. Rev. D 46, 5346 (1992).

[16] G. Field and S. Carroll, Phys. Rev. D 62, 103008 (2000); M. Giovannini, Phys. Rev. D 61, 063502 (2000); 61, 063004 (2000); K. Bamba, Phys. Rev. D 74, 123504 (2006); K. Bamba, C. Q. Geng, and S. H. Ho, Phys. Lett. B 664, 154 (2008).

[17] L. Campanelli, Int. J. Mod. Phys. D 18, 1395 (2009); L. Campanelli and M. Giannotti, Phys. Rev. D 72, 123001 (2005); Phys. Rev. Lett. 96, 161302 (2006); M. Giovannini, Phys. Rev. D 88, 063536 (2013); K. Bamba, Phys. Rev. D 91, 043509 (2015); L. Campanelli, Eur. Phys. J. C 75, 278 (2015).

[18] M. Giovannini and M. E. Shaposhnikov, Phys. Rev. D 57, 2186 (1998); Phys. Rev. Lett. 80, 22 (1998); M. Giovannini, Phys. Rev. D 61, 063004 (2000).

[19] M. Giovannini, Phys. Rev. D 61, 063502 (2000); M. Dvornikov and V. B. Semikoz, J. Cosmol. Astropart. Phys. 02 (2012) 040; S. Alexander, A. Marciano, and D. Spergel, J. Cosmol. Astropart. Phys. 04 (2013) 046; N. D. Barrie and A. Kobakhidze, J. High Energy Phys. 09 (2014) 163.

[20] D. E. Kharzeev, Prog. Part. Nucl. Phys. 75, 133 (2014); M. Giovannini, Phys. Rev. D 88, 063536 (2013); N. Yamamoto, arXiv: 1505.05444.

[21] D. Kharzeev, L. McLerran, and H. Warringa, Nucl. Phys. A803, 227 (2008); K. Fukushima, D. Kharzeev, and H. Warringa, Phys. Rev. D 78, 074033 (2008); D. Kharzeev, Ann. Phys. (Amsterdam) 325, 205 (2010).

[22] M. Giovannini, Phys. Rev. D 88, 083533 (2013); 89, 063512 (2014).

[23] G. Feinberg and J. Sucher, Phys. Rev. A 2, 2395 (1970); Phys. Rev. D 20, 1717 (1979).

[24] S. Deser and C. Teitelboim, Phys. Rev. D 13, 1592 (1976); S. Deser, J. Phys. A 15, 1053 (1982); M. Giovannini, J. Cosmol. Astropart. Phys. 04 (2010) 003.

[25] M. Giovannini, Phys. Rev. D 86, 103009 (2012); 87, 083004 (2013); Classical Quantum Gravity 21, 4209 (2004).

[26] A. R. Liddle and S. M. Leach, Phys. Rev. D 68, 103503 (2003). 